\title{
Wie normativ ist die Kommunikationswissenschaft?
}

\section{Ein inhaltsanalytischer Vergleich deutscher und US-amerikanischer Fachzeitschriftenaufsätze}

\author{
Stephanie Geise - Ulrike Klinger - Melanie Magin • \\ Kathrin Friederike Müller • Cordula Nitsch • Claudia Riesmeyer • \\ Liane Rothenberger • Christina Schumann • Annika Sehl • \\ Cornelia Wallner • Arne Freya Zillich
}

Eingegangen: 27. April 2020 / Angenommen: 30. Juli 2020 / Online publiziert: 26. Januar 2021

(C) Der/die Autor(en) 2021

Alle Autorinnen haben gleichermaßen zur Publikation beigetragen.

Zusatzmaterial online Zusätzliche Informationen sind in der Online-Version dieses Artikels (https:// doi.org/10.1007/s11616-021-00638-3) enthalten.

PD Dr. S. Geise

Institut für Kommunikationswissenschaft, Westfälische Wilhelms-Universität Münster, Münster, Deutschland

E-Mail: stephanie.geise@uni-muenster.de

Prof. Dr. U. Klinger

Professur für Politische Theorie und Digitale Demokratie, Europa-Universität Viadrina Frankfurt (Oder), Frankfurt (Oder), Deutschland

E-Mail: klinger@europa-uni.de

Assoc. Prof. Dr. M. Magin

Norwegian University of Science and Technology (NTNU), Trondheim, Norwegen

E-Mail: melanie.magin@ntnu.no

Dr. K. F. Müller

Institut für Medienforschung, Universität Rostock, Rostock, Deutschland

E-Mail: kathrin.mueller@uni-rostock.de

Dr. C. Nitsch

Professur für Kommunikationswissenschaft - Öffentliche Kommunikation, Universität Augsburg, Augsburg, Deutschland

E-Mail: cordula.nitsch@phil.uni-augsburg.de

Dr. C. Riesmeyer · Dr. C. Wallner

Institut für Kommunikationswissenschaft und Medienforschung, Ludwig-Maximilians-Universität München, München, Deutschland

Dr. C. Riesmeyer

E-Mail: riesmeyer@ifkw.lmu.de

Dr. C. Wallner

E-Mail: Cornelia.Wallner@ifkw.lmu.de 
Zusammenfassung Kommunikationswissenschaftliche Forschung ist durch die strukturellen Rahmenbedingungen geprägt, unter denen sie entsteht - etwa durch das Wissenschaftssystem, die gesellschaftlichen Erwartungen an Wissenschaft und das Mediensystem des jeweiligen Landes. Die quantitative Inhaltsanalyse untersucht im Vergleich zwischen Deutschland und den USA, inwiefern sich solche Rahmenbedingungen auf die normativen Vorstellungen auswirken, die Autorinnen aus diesen Ländern in ihren Zeitschriftenaufsätzen zum Ausdruck bringen. Hierzu wenden wir ein dreistufiges Vorgehen zur Identifikation, Systematisierung und Kategorisierung von „Sollensvorstellungen“ an und schlagen damit erstmals ein methodisches Verfahren vor, mit dem sich die Normativität von Fachzeitschriftenaufsätzen messen lässt, ohne die zu erfassenden Werte und Normen vorab festzulegen. Indikator für die Normativität der Aufsätze sind die darin artikulierten Sollensvorstellungen und konkreten Handlungsempfehlungen, die sich an verschiedene Gruppen richten können. Die Befunde zeigen keinen Unterschied im Grad der Normativität, wohl aber in den Schwerpunkten, die dabei gesetzt werden: Sollensvorstellungen und Handlungsempfehlungen in den deutschen Aufsätzen konzentrieren sich stärker auf Medien und Journalismus als auf unmittelbare Forschungsgegenstände des Fachs. Die USamerikanischen Aufsätze hingegen weisen mit Schwerpunkt auf einem gelungenen gesellschaftlichen Zusammenleben stärker über das Fach hinaus und appellieren häufiger an die Verantwortung individueller Akteurinnen. Die Ergebnisse machen deutlich, dass strukturelle Unterschiede - auch vermittelt über die Forschungsgegenstände - die normativen Vorstellungen prägen, die Wissenschaftlerinnen leiten und von ihnen geäußert werden. Damit ruft die Studie die normative Prägung der Kommunikationswissenschaft ins Bewusstsein und liefert neue Erkenntnisse über das Selbstverständnis des Fachs.

Schlüsselwörter Normativität · Kommunikationswissenschaft ·

Sollensvorstellungen · Handlungsempfehlungen · Inhaltsanalyse · Vergleichende Forschung

PD Dr. L. Rothenberger · Dr. C. Schumann

Institut für Medien und Kommunikationswissenschaft, Technische Universität Ilmenau, Ilmenau, Deutschland

PD Dr. L. Rothenberger

E-Mail: liane.rothenberger@tu-ilmenau.de

Dr. C. Schumann

E-Mail: Christina.Schumann@tu-ilmenau.de

Prof. Dr. A. Sehl

Professur für Digitalen Journalismus, Universität der Bundeswehr München, München, Deutschland E-Mail: annika.sehl@unibw.de

Dr. A. F. Zillich $(\bowtie)$

Digitale Medienkultur, Filmuniversität Babelsberg KONRAD WOLF,

Marlene-Dietrich-Allee 11, 14482 Potsdam, Deutschland

E-Mail: a.zillich@filmuniversitaet.de 


\section{How normative are communication studies?}

A comparative content analysis of German and US-American journal articles

Abstract Research in communication studies is shaped by the structural conditions under which it is conducted-for example, the science system, societal expectations of science, and the media system of the respective country. The USA are often seen as a reference frame for the development of the German Communication Studies, but differ structurally from Germany in terms of its science, society, and media system. Therefore, the study at hand compares Germany with the USA and examines the extent to which such structures affect the normative ideas that authors from these countries express in their journal articles. The normativity of the articles is measured by the normative claims and concrete calls for action articulated in them towards different groups of addressees. Normative claims exist in two variants: they can evaluate a current condition or articulate a desirable condition for the future.

To answer our research question "Does the normativity of communication studies, articulated in journal articles, differ between Germany and the USA?", we conducted a quantitative content analysis of communication studies articles published between 1970 and 2014 in scientific journals of our subject. The period under study thus encompasses a period of older and more recent intensive upheavals in society, science, and media systems (e.g., changing values, dualization of European broadcasting systems, digitalization), which challenge the working conditions and research subjects of communication scholars and are therefore likely to evoke normative claims. Given our interest in the normative statements of German and US-American communication scholars, we selected articles with at least one author with German affiliation from three German journals (German sample) and articles with at least one author with US affiliation from the 20 international journals (US sample). This results in a sample of 326 articles $\left(\mathrm{n}_{\text {German }}=61, \mathrm{n}_{\mathrm{US}}=265\right)$, in which 3233 normative claims $\left(\mathrm{n}_{\mathrm{German}}=691, \mathrm{n}_{\mathrm{US}}=2542\right)$ were identified. For each normative claim, the content, the addressed subject and object as well as calls of action, the type of normative claim and the mentioned research field were coded by 12 communication researchers.

The results confirm the strong normative influence of both German and USAmerican communication studies: almost nine out of ten articles contain normative claims. Regarding further formal indicators the two countries hardly differ: In both German and US-American articles desirable conditions for the future outweigh evaluations of current conditions. The majority of the authors themselves take a normative position instead of citing statements by other authors. Only about every third normative claim in the articles of both countries names a subject that is supposed to create the desirable state, every fifth claim addresses an object that is supposed to profit from this state, and every third claim is connected with a recommendation for action.

These findings do not point to a different degree of normativity, but clearly indicate different foci of normativity: The normative claims and calls for action in the German articles focus strongly on media and journalism as main research subjects of the discipline. The US-American articles, on the other hand, focus strongly on successful social coexistence, thus pointing more beyond the discipline's main subject, and appeal more often to the responsibility of individual actors. The re- 
sults indicate that structural differences-also conveyed through the subjects of research-decisively shape the normative ideas that guide communication scholars and are expressed by them. The study thus raises awareness of the normative character of communication studies and contributes to a better self-understanding of the discipline.

The present study extends preliminary work on the normativity of communication studies in several respects: Firstly, the proposed instrument of normative claims and call for action provides an opportunity to make normativity visible independently from the concrete use of the terms "norms" and "values"-in scientific publications and beyond. Secondly, the methodological design developed for the present study enables not only systematic comparisons across individual scientific sub-areas or research subjects, but also between different academic cultures. Thirdly, the concept of manifestations of normativity makes an important contribution, which can serve the self-reflection of communication studies and its normativity: The comparison elucidates how strongly one's scientific work is shaped by the surrounding structures. Which research topics are perceived as relevant and which normative positions are represented strongly depends on the national and cultural context in which communication scholars are scientifically socialized and work.

Keywords Normativity - Communication Studies · Normative Claims - Guidance · Content Analysis · Comparative Research

\section{Einleitung}

Wissenschaftlichem Handeln liegen bewusst oder unbewusst Normen und Werte zugrunde (vgl. Anderson et al. 2010, S. 366). Sie bestimmen Handlungsregeln und ethische Grenzen für Wissenschaftlerinnen ${ }^{1}$ (vgl. Braxton 2010, S. 243; Bruhn 2008, S. 19), schlagen sich in Routinen und sozialen Praktiken sowie in Ethikkodizes von Fachgesellschaften nieder und beeinflussen, wie Wissenschaftlerinnen lehren und forschen. Im Forschungsprozess sind zahlreiche Entscheidungen normativ geprägt von der Wahl der Forschungsfragen über Theorien und Methoden bis zur Interpretation der Ergebnisse (vgl. Althaus 2012, S. 99-100; Scherer 2013, S. 254-261; Zillich et al. 2016, S. 395). Normen und Werte leiten Wissenschaftlerinnen an, Fragestellungen zu untersuchen, die sie als gesellschaftlich relevant und aktuell empfinden, und beeinflussen, wann und zu welchen Themen sie ihre Forschungsergebnisse in den öffentlichen Diskurs einbringen (vgl. Bruhn 2008, S. 20; Peters 2019, S. 209; für Beispiele siehe Öffentliche Kommunikationswissenschaft 2019) und so die gesellschaftliche Legitimation ihres Fachs sichern (vgl. Eberwein und Fengler 2012, S. 12).

Neben internalisierten Normen und Werten, die von der Mehrheit der Wissenschaftlerinnen unabhängig von ihrem fachlichen Hintergrund geteilt werden (vgl. Merton 1973, S. 269), beeinflussen spezifische Rahmenbedingungen innerhalb und

\footnotetext{
1 Aus Gründen der besseren Lesbarkeit wird im Text das generische Femininum verwendet. Diese Form referiert auf Personen aller biologischen und sozialen Geschlechter.
} 
außerhalb des Wissenschaftssystems, wie Wissenschaftlerinnen arbeiten können und sollen (vgl. Löblich 2010a, S. 39). Beispielsweise wird am Anfang einer wissenschaftlichen Karriere fach- und länderspezifisches ,tacit knowledge“(Gerholm 1990, S. 268) durch Interaktionen mit Kolleginnen erworben. Auch die strukturellen und finanziellen Bedingungen des jeweiligen Wissenschaftssystems (vgl. Vogler und Post 2019, S. 320), gesellschaftliche und politische Erwartungen an Wissenschaftlerinnen sowie - im Fall der Kommunikationswissenschaft - das nationale Mediensystem (vgl. Löblich 2010a, S. 549-550) beeinflussen die Tätigkeit von Wissenschaftlerinnen. Diese Rahmenbedingungen beeinflussen ihre Forschungsgegenstände und damit vermutlich auch ihre normativen Äußerungen in wissenschaftlichen Publikationen. Untersuchungen der strukturellen Einflüsse auf die Normativität wissenschaftlicher Publikationen können insofern zum Selbstverständnis der Kommunikationswissenschaft beitragen, fehlen bislang aber weitgehend.

Die nachfolgende Studie untersucht, welche Normen und Werte deutsche und US-amerikanische Forschende in wissenschaftlichen Publikationen von 1970 bis 2014 artikulieren. ${ }^{2}$ Die USA werden oft als Referenzrahmen der hiesigen Fachentwicklung gesehen (vgl. Beck 2020, S. 169; Löblich 2010a, S. 129), unterscheiden sich aber hinsichtlich des Wissenschafts-, Gesellschafts- und Mediensystems von Deutschland. Daher vergleicht die Studie normative Äußerungen von Autorinnen aus Deutschland und den USA, mit denen sie selbst oder mit Bezug auf andere Quellen gegenwärtige Zustände bewerten oder als erstrebenswerte Zustände bezeichnen. Mit Sollensvorstellungen und Handlungsempfehlungen schlägt die Studie eine neuartige Operationalisierung der Normativität wissenschaftlicher Texte vor. Ihre Ergebnisse können als Ausgangspunkt dienen, um das normative Verständnis des Fachs zu reflektieren.

Der Aufsatz beginnt mit einem Vergleich relevanter Rahmenbedingungen der deutschen und US-amerikanischen Kommunikationswissenschaft. Anschließend beschreiben wir, wie sich Normativität in wissenschaftlichen Publikationen manifestieren kann, stellen die Methode und Befunde unserer Studie vor und diskutieren ihre Implikationen für die Kommunikationswissenschaft und ihr Selbstverständnis.

\section{Einflüsse auf und Messung von Normativität in der Kommunikationswissenschaft}

\subsection{Wissenschaftliche Normen und Werte}

Normen sind geteilte Vorstellungen einer sozialen Gruppe an ein erwartetes oder wünschenswertes Verhalten in einer bestimmten Situation (vgl. Braxton 2010, S. 243). Werte umfassen ,,Vorstellungen vom Wünschenswerten“ (Schäfers 2016,

\footnotetext{
2 Diese Studie präsentiert Befunde des von der Deutschen Forschungsgemeinschaft finanzierten Netzwerks „Werte und Normen als Forschungsgegenstände und Leitbilder in der Kommunikationswissenschaft" (ZI 1543/1-1). An der Konzeption der Studie und der Erhebung der Daten beteiligten sich Stephanie Geise, Katharina Kleinen-von Königslöw, Ulrike Klinger, Melanie Magin, Kathrin Friederike Müller, Cordula Nitsch, Claudia Riesmeyer, Liane Rothenberger, Christina Schumann, Annika Sehl, Cornelia Wallner und Arne Freya Zillich gleichermaßen.
} 
S. 39), die auf kulturellen, religiösen, ethischen und sozialen Leitbildern beruhen: „Werte sind nicht vorausgesetzt, sondern abgeleitet.“ (Sommer 2016, S. 16) Beide Konzepte sind eng miteinander verbunden und lassen sich analytisch schwer trennen: Idealtypisch stellen Werte übergeordnete Orientierungsstandards für Denken und Handeln bereit. Normen liefern die entsprechenden kontextabhängigen Handlungsregeln (vgl. Schäfers 2016, S. 39) und fungieren als Bindeglied zwischen dem Wünschenswerten und dem Praktischen (vgl. Schicha 2010, S. 23). Sowohl Normen als auch Werte haben eine ,intersubjektive Gültigkeit für einen bestimmten Zeitraum innerhalb einer bestimmten Kultur" (Schicha 2010, S. 22). Im Unterschied dazu stellen Prinzipien übergreifende Regeln dar, die als allgemein gültig vorausgesetzt werden und somit einen universalistischen Anspruch haben (vgl. Rath 2010, S. 137; Schicha 2010, S. 22). Beispiele für solche Prinzipien sind Autonomie, Nichtschaden, Wohltun und Gerechtigkeit (vgl. Beauchamp und Childress 2013, S. 13). Im Zusammenspiel von Prinzipien, Werten und Normen entsteht Normativität als Konsens, der nach Kuhn als ein Paradigma der Wissenschaft gilt: „Alles, worin die Wissenschaftler einer Disziplin übereinstimmen bzw. übereinstimmen können“, bildet ein Paradigma (Kuhn 1962, S. 37). Nur Wissenschaftlerinnen, die einem Paradigma folgen, können Wissenschaft gestalten und neue Befunde generieren (vgl. Lang 2013, S. 10). Ein Konsens über von Wissenschaftlerinnen geteilte Normen und Werte kann daher leitend für ihr wissenschaftliches Handeln sein.

Analysen dieses normativen Handelns gewinnen in der Kommunikationswissenschaft in jüngerer Zeit zunehmend an Bedeutung (vgl. Blumler und Cushion 2014; Christians et al. 2009; Heesen 2016; Kepplinger 2014; Neuberger 2017a). Sie veranschaulichen, dass sich normative Äußerungen von Wissenschaftlerinnen als Ausdruck des Handelns auf unterschiedliche Begründungsebenen beziehen können, je nachdem, ob es um universalistische Prinzipien der Idealebene, sozio-kulturelle Werte und Normen der Praxisebene oder individuelle Interessen geht (vgl. Schicha 2010, S. 33-34). So gründen beispielsweise zahlreiche Öffentlichkeitstheorien auf den Prinzipien politische Rationalität und Gerechtigkeit (vgl. Kepplinger 2014, S. 25-31), und normative Begründungen des gesellschaftlichen Auftrags von Massenmedien thematisieren oft das Prinzip des Gemeinwohls (vgl. Serong 2011, S. 107-112). Analysen zur Qualität öffentlicher Kommunikation hingegen beziehen sich häufig auf Werte wie Freiheit, Gleichheit, Vielfalt oder Integration (vgl. McQuail 1992, S. 65-80; Neuberger 2017a, S. 51-52) und Überlegungen zur journalistischen Objektivität auf den Wert Wahrheit (vgl. Neuberger 2017b, S. 407). Empirisch lässt sich ermitteln, inwieweit massenmediale Angebote diesen Wert für die Gesellschaft oder die Individuen erzielen (vgl. Hasebrink 2016, S. 27-31).

Gleichzeitig gilt es, den logischen Widerspruch von empirischen Prämissen und normativen Schlussfolgerungen zu beachten, der auf die grundlegende Differenz von Sein und Sollen verweist (vgl. Rath 2010, S. 136-138). Aus empirischen Aussagen, die sich in Seinssätzen ausdrücken lassen, folgen logisch keine Sollenssätze, sprich normative Äußerungen. „Wird eine Aussage deskriptiv oder empirisch verstanden, die nicht deskriptiv oder empirisch ist" (Rath 2010, S. 137), liegt ein naturalistischer Fehlschluss vor. Während es folglich unproblematisch ist, dass sich Wissenschaftlerinnen bei der Themenwahl und Theoriebildung von Normen und Werten leiten lassen (Entdeckungszusammenhang), sie zum Gegenstand ihrer Forschung machen 
(vgl. Albert 1993, S. 204) und die gesellschaftlichen und ethischen Konsequenzen ihres wissenschaftlichen Tuns reflektieren (Verwertungszusammenhang), ist es nicht die Aufgabe der Wissenschaft, ,,praktische Werturteile als wissenschaftliche Annahmen auszugeben“ (Dahrendorf 1968, S. 84). Die geforderte Werturteilsfreiheit der Wissenschaft bedeutet folglich nicht, dass Wissenschaftlerinnen keine Empfehlungen aussprechen können, wie das angestrebte Ziel verwirklicht werden kann. Vielmehr zielt sie darauf ab, Werturteile als Begründungszusammenhang von Forschung zu vermeiden, wissenschaftliche Aussagen intersubjektiver Kritik zu unterziehen und die Umsetzung der ausgesprochenen Empfehlungen Praktikerinnen, Politikerinnen und Juristinnen zu überlassen (vgl. Albert 1993, S. 205; Dahrendorf 1968, S. 86; Joas 1997, S. 14).

\subsection{Rahmenbedingungen wissenschaftlicher Normen und Werte}

Normen und Werte sind kultur- und zeitspezifisch (vgl. Schicha 2010, S. 22). Daher ist $\mathrm{zu}$ erwarten, dass Kommunikationswissenschaftlerinnen verschiedener Herkunft unterschiedliche normative Positionen vertreten und sich dies auch in ihren Publikationen spiegelt. Im Folgenden werden relevante Unterschiede zwischen den Wissenschaftssystemen, den gesellschaftlichen Erwartungen an Wissenschaft und den Mediensystemen in Deutschland und den USA als Rahmenbedingungen beschrieben (vgl. Löblich und Scheu 2011, S. 7), die solche normativen Positionen beeinflussen dürften.

\subsubsection{Rahmenbedingungen im Wissenschaftssystem}

Relevant sind im Wissenschaftssystem individuelle und strukturelle Faktoren (vgl. Post 2013, S. 28-49.), die sich wechselseitig beeinflussen: Auf individueller Ebene formulieren Wissenschaftlerinnen Ansprüche an das eigene Handeln, die sie im Laufe ihrer wissenschaftlichen Sozialisation internalisieren (vgl. Anderson et al. 2010, S. 390; Gerholm 1990, S. 263). Hierdurch definieren sie, wie sie zur wissenschaftlichen Erkenntnis beitragen wollen (vgl. Peters 2019, S. 211). Dass viele Wissenschaftlerinnen dies gleichermaßen tun, sichert der Gesellschaft ,überdauernde Erkenntnis und verlässliches Verwertungswissen zu“ (Post 2013, S. 47) und rechtfertigt den gesellschaftlichen Stellenwert von Wissenschaft. Internalisierte Ansprüche an das eigene wissenschaftliche Handeln werden z. B. im unterschiedlichen Fachverständnis in Deutschland und den USA deutlich. Ein Fachverständnis ,hat immer auch eine normative Funktion“ (Löblich 2010b, S. 545). Das Fachverständnis in den USA prägten interdisziplinäre Zugänge und Fachkulturen; die ,heavy hitters“ (Herbst 2008, S. 604) der Kommunikationswissenschaft stammten aus der Soziologie, der Politikwissenschaft und den Wirtschaftswissenschaften, die sich in den Anfängen aktuellen gesellschaftlichen Kommunikationsphänomen mit dem Ziel widmeten, diese besser zu verstehen - wie Fragen der Medienwirkungsforschung bzw. der soziologischen, politischen und psychologischen Rolle von Propaganda (vgl. Soffer und Geifman 2020, S. 4; Waisbord 2016, S. 875). Zudem prägten kulturwissenschaftliche Ansätze (u. a. durch die emigrierten Wissenschaftler der Frankfurter Schule) sowie ,,interpersonal relations, speech rhetoric studies, and a focus on 
communication organizations" die Anfänge des US-amerikanischen Fachs (Soffer und Geifman 2020, S. 4). Diese inhaltliche Vielfalt kennzeichnet das Fach bis heute, das nach Waisbord (2016, S. 868) inzwischen Tendenzen einer fragmentierten, inhaltlich spezifizierten Kommunikationswissenschaft zeigt.

Das empirisch-sozialwissenschaftliche Verständnis der deutschen Kommunikationswissenschaft (vgl. DGPuK 2008, S. 2) setzte sich hingegen in der Mitte des 20. Jahrhunderts als Reaktion auf verschiedene Veränderungen in der Umwelt des Fachs durch (vgl. Löblich 2010a, 2010b). Neue Medienstrukturen, die aus der Einführung des Fernsehens, der Konzentration auf dem Pressemarkt und dem steigenden Bedarf an unbelasteten Journalistinnen nach dem Ende des Zweiten Weltkriegs resultierten, veränderten zum einen die Forschungsgegenstände der damaligen Publizistik- und Zeitungswissenschaft. Zum anderen erhöhte die wachsende Bedeutung der Massenmedien für politische und unternehmerische Entscheidungen bei Themen wie Vielfalt und Meinungsmacht die Nachfrage nach quantitativen, unmittelbar verwertbaren Daten. Um als Auftragnehmer für die nachgefragten empirischen Studien berücksichtigt und in beratende Gremien aufgenommen zu werden, orientierte sich das Fach stärker an empirisch-sozialwissenschaftlichen Forschungsgegenständen und Methoden (vgl. Löblich 2010b, S. 549-550). Historische und literaturwissenschaftliche Untersuchungen verloren dadurch sukzessive an Bedeutung (vgl. Beck 2020, S. 169). Zur „empirisch-sozialwissenschaftlichen Wende“ (vgl. Löblich, 2010a, 2010b) trugen daneben die wachsende Orientierung an den Nachbardisziplinen Soziologie und Politikwissenschaft und die Vorreiterrolle der US-amerikanischen Mass Communication Research bei, die sich vorrangig auf analytische Wissenschaftstheorien und quantitative Methoden stützten (vgl. Löblich 2010b, S. 550). An deutschen Instituten mit sozialwissenschaftlicher Orientierung beeinflusste diese Ausrichtung an der US-amerikanischen Kommunikationsforschung auch die Lehre und das normative Wissenschaftsverständnis nachfolgender Generationen von deutschen Kommunikationswissenschaftlerinnen (vgl. Löblich, 2010b, S. 553-554).

Da die Kommunikationswissenschaft durch eine Vielzahl an fachgeschichtlichen Zugängen und inhaltlichen Ausrichtungen geprägt ist, liegt die Annahme nahe, dass sich diese strukturellen Rahmenbedingungen in den individuellen Normen und Werten von Wissenschaftlerinnen widerspiegeln und sich ggf. von Nation zu Nation unterscheiden (vgl. Averbeck-Lietz 2012, S. 6-7). Individuelle Normen und Werte werden zudem durch wissenschaftsinterne Mechanismen der Qualitätssicherung beeinflusst. Beispielsweise ist in der Kommunikationswissenschaft mittlerweile sowohl in den USA als auch in Deutschland das Peer-Review-Verfahren von Publikationen und Tagungen zur Qualitätssicherung etabliert (vgl. Gehrau et al. 2013, S. 323; Post 2013, S. 28-49), wobei dieser Mechanismus in den USA eine längere Tradition hat. Außerdem beeinflussen zeitliche und finanzielle Ressourcen des wissenschaftlichen Arbeitens die individuellen Normen und Werte von Forschenden. Zum Beispiel ist in Deutschland der Großteil der Hochschulen öffentlich finanziert und kann weitgehend autonom über seine Fragestellungen entscheiden (vgl. Post 2013, S. 32). Neben dieser Finanzierung sind die wichtigsten Geldgeber öffentliche Institutionen wie die Deutsche Forschungsgemeinschaft oder Ministerien, die auch Grundlagenforschung fördern. In den USA hingegen sind privat finanzierte Hochschulen sehr verbreitet - und mit ihnen eine ,marktförmige Instrumentalisierung der Bildung“ 
(Lenhardt 2005, S. 16). US-amerikanische Universitäten sind an ,ökonomischen Prämissen ausgerichtet“" (Harnischmacher 2010, S. 94) und stehen im Wettbewerb um Ressourcen untereinander. Als relevante Stakeholder bestimmen dort die Geldgeberinnen in weitaus stärkerem Maß über die Forschungsthemen mit (vgl. Vogler und Post 2019, S. 334).

\subsubsection{Gesellschaftliche Erwartungen an Wissenschaft}

Auch die Gesellschaft richtet allgemeine Erwartungen an ,gute Wissenschaft“ und definiert durch den öffentlichen Diskurs relevante Themen, zu denen Wissenschaftlerinnen arbeiten sollten (aktuell z. B. Klimawandel, Coronakrise). Die wissenschaftlich fundierten Antworten und Prognosen, die gesellschaftliche Stakeholder dabei erwarten, können die wissenschaftliche Themensetzung (vgl. Kepplinger und Post 2008, S. 25) und sogar die Etablierung ganzer Forschungsfelder bestimmen. So bildete sich die Gesundheitskommunikation Ende der 1980er-Jahre als Forschungsfeld in den USA heraus, während sie sich in Deutschland bis in die 2000er-Jahre noch im Anfangsstadium befand (vgl. Schulz und Hartung 2010, S. 548). Zudem haben in der US-amerikanischen Fachtradition vor allem anwendungsbezogene Forschungsprojekte Erfolg, die ,,politische und wirtschaftliche Entscheidungen legitimieren oder vielleicht sogar beeinflussen können“ (Meyen 2013, S. 130) und so für Reputationsgewinn sorgen.

\subsubsection{Rahmenbedingungen im Mediensystem}

Während Wissenschaftssystem und Gesellschaft disziplinübergreifend Einfluss nehmen, wird die Kommunikationswissenschaft fachspezifisch auch durch das Mediensystem normativ geprägt. Die Mediensysteme beider hier betrachteten Länder unterscheiden sich gemäß der Typologie von Hallin und Mancini (2004, S. 67) deutlich. Deutschland hat ein demokratisch-korporatistisches Mediensystem, die USA ein liberales. Die strukturellen Unterschiede der Mediensysteme können auf vielfältige Weise beeinflussen, welche Forschungsgegenstände Kommunikationswissenschaftlerinnen als relevant empfinden und welche normativen Vorstellungen sie in diesem Zusammenhang äußern:

1. In der Zeitungsindustrie beider Länder entwickelte sich früh eine Massenpresse, wobei die Zeitungsauflagen in den USA bis heute deutlich geringer sind als in Deutschland (vgl. Hallin und Mancini 2004, S. 67). Dieser strukturelle Unterschied könnte sich z.B. in der Bedeutsamkeit von Zeitungen als Forschungsgegenstand und der normativen Bewertung ihres gesellschaftlichen Stellenwertes widerspiegeln.

2. Der politische Parallelismus - das Ausmaß, in dem das Mediensystem die ideologischen Strukturen des politischen Systems reflektiert - war in Deutschland historisch stark ausgeprägt, weil die Entwicklung der Zeitungen eng mit der Entwicklung der politischen Parteien verknüpft war. Im Laufe unseres Untersuchungszeitraums nahm er deutlich ab, ist bis heute aber erheblich stärker als in den USA, wo sämtliche Medienmärkte von Beginn an stark kommerzialisiert waren (vgl. Magin 
im Druck). Dieser Unterschied spielt normativ z.B. für Studien eine Rolle, die sich mit journalistischer Parteilichkeit oder dem (originär angloamerikanischen) Gebot der Trennung von Nachricht und Meinung befassen.

3. Die journalistische Professionalisierung hat in den USA eine längere Tradition als in Deutschland, sichtbar z. B. an der früheren Etablierung journalistischer Selbstkontrollorgane (vgl. Redelfs 1996, S. 190-191) und der stärkeren Akademisierung des Journalistenberufs (vgl. Harnischmacher 2010, S. 270) in den USA. Normativ beeinflusst dies z.B. Untersuchungen zu journalistischer Ethik, journalistischen Fehlleistungen, Journalistinnenausbildung oder zum Rollenverständnis von Journalistinnen.

4. Auch die Rolle des Staates im Mediensystem unterscheidet sich: Die deutsche Medienpolitik ist im Vergleich zu den USA stärker interventionistisch und zielt besonders auf die Sicherung von Pressefreiheit, Medienvielfalt und journalistischer Autonomie ab. Eines der wichtigsten Instrumente hierzu ist der öffentlich-rechtliche Rundfunk. In den USA hingegen geht die Medienpolitik von der Selbstregulierung der Medienmärkte aus und verhält sich entsprechend zurückhaltend. Der öffentlich-rechtliche Rundfunk spielt kaum eine Rolle (vgl. Brüggemann et al. 2014, S. 1041). Das dürfte z. B. zu einem größeren Stellenwert der an den öffentlich-rechtlichen Rundfunk gerichteten normativen Erwartungen in der deutschen kommunikationswissenschaftlichen Forschung führen.

\subsection{Sollensvorstellungen und Handlungsempfehlungen als Indikatoren von Normativität}

Inwiefern diese unterschiedlichen Rahmenbedingungen tatsächlich die normativen Positionen deutscher und US-amerikanischer Autorinnen prägen, können neben Befragungen von Wissenschaftlerinnen (vgl. Anderson et al. 2010) systematische Inhaltsanalysen kommunikationswissenschaftlicher Publikationen klären. Entsprechende Studien dazu gibt es bisher aber kaum. Werden Normen und Werte inhaltsanalytisch untersucht, dann meist bezogen auf einzelne Themen (vgl. Boudana 2011; Singer et al. 2011), Medieninhalte (vgl. Chesebro 2003; Foss 2008) oder Teilbereiche des Fachs (vgl. Fink und Gantz 1996), nicht aber im Vergleich verschiedener Fachkulturen. Daher gilt es, zunächst geeignete Indikatoren für die (vergleichende) Messung von Normativität zu entwickeln. Die naheliegende Möglichkeit, die in den Texten explizit genannten Normen und Werte zu erfassen (vgl. z. B. Fink und Gantz 1996), ist aufgrund der mangelnden Trennschärfe beider Konzepte (Kapitel 2.1) jedoch schwierig und wird durch ein unterschiedliches Begriffsverständnis in verschiedenen Disziplinen verstärkt (vgl. Zillich et al. 2016, S. 398-399).

Eine Lösung bietet das analytische Konzept der Sollensvorstellungen (vgl. Boventer 1984, S. 279; Thomaß 2016, S. 33), das Normen und Werte gleichermaßen umfasst und für die vorliegende empirische Analyse der Normativität wissenschaftlicher Publikationen genutzt wird. Sollensvorstellungen werden hier in Anlehnung an Esser (2000) als normative Aussagen verstanden, in denen eine Erwartung an eine Handlung zu Ansprüchen zusammengeführt wird. Erwartungen können beispielsweise Anreize zu einer Handlung oder die Folgen (auch mögliche Sanktionen) einer 
Handlung beinhalten. Ansprüche sind Erwartungen, die bewertet werden und deshalb verbunden sind mit „Wertungen, Wünschen, Forderungen“ (Esser 2000, S. 75). Esser (2000, S. 51-55) zufolge besteht eine Sollensvorstellung aus drei Elementen: (1) Der Inhalt ist die Erwartung, die in der Sollensvorstellung zum Ausdruck kommt (was soll geschehen/getan werden?). (2) Das Subjekt ist die Adressatin, die für die Realisierung dieser Erwartung verantwortlich gemacht wird (wer soll die Initiative ergreifen?). (3) Das Objekt ist die „Nutznießerin“, die von der Realisierung der Erwartung betroffen ist (für wen/was trägt das Subjekt Verantwortung?). Die Sollensvorstellung „Eltern sollen den Medienkonsum ihrer Kinder kontrollieren“ besteht aus dem Inhalt „den Medienkonsum kontrollieren“, dem Subjekt „Eltern“ und dem Objekt ,ihre Kinder““.

Mit dieser Dreiteilung bietet das Konstrukt der Sollensvorstellung den entscheidenden Vorteil, dass es inhaltsanalytisch besser operationalisierbar ist als die Konstrukte Normen und Werte. Der Inhalt ist das zentrale Element jeder Sollensvorstellung. Er muss zwingend vorhanden sein, damit eine Sollensvorstellung vorliegt, während ein Subjekt und/oder ein Objekt vorhanden sein kann, aber nicht muss. Die Sollensvorstellung ,die dringende Forderung nach einer vielfältigen Medienlandschaft“ (Inhalt: vielfältige Medienlandschaft) enthält z. B. weder Subjekt noch Objekt (für weitere Beispiele siehe die Schlüsselpläne im zusätzlichen Anhang der Online-Version dieses Aufsatzes). Sollensvorstellungen können in zwei Formen vorkommen: (1) Sie können einen gegenwärtigen Zustand explizit bewerten und beschreiben, dass etwas positiv/gut bzw. negativ/schlecht ist. In dieser Bewertung kommt ein gegenwärtiger Anspruch zum Ausdruck. (2) Sie können einen zukünftigen Zustand als erstrebenswert beschreiben und einen zukünftigen Anspruch zum Ausdruck bringen, wie etwas (nicht) sein sollte (vgl. Esser 2000, S. 74-75).

Noch einen Schritt weiter gehen Wissenschaftlerinnen, wenn sie aus Sollensvorstellungen explizite Handlungsempfehlungen ableiten. Gemeint sind damit konkrete Anleitungen oder Ratschläge, wie bestimmte Adressatinnen(gruppen) - etwa Politik, Medien, Unternehmen oder Nutzerinnen - unter bestimmten Bedingungen oder generell handeln sollen. Sie richten sich an mindestens eine klar erkennbare, explizit benannte Adressatin und benennen konkrete künftige Aktivitäten der Adressatinnen, um einen wünschenswerten Zustand herzustellen.

\subsection{Sichtbarkeit von Normativität in wissenschaftlichen Texten}

Anhand der Sollensvorstellungen und ihrer unterschiedlichen Manifestationen lässt sich die Normativität wissenschaftlicher Publikationen messen. Im Folgenden beschreiben wir die Indikatoren, anhand deren wir die Fachkulturen in Deutschland und den USA vergleichen, und leiten daraus unsere Forschungsfragen ab. Abb. 1 illustriert, wie in diesen Indikatoren eine immer stärkere bzw. explizitere Manifestation von Normativität zum Ausdruck kommt. Wir operationalisieren Manifestationen von Normativität als Sollensvorstellungen in wissenschaftlichen Fachzeitschriftenaufsätzen, deren Zielgruppe in erster Linie Forschende derselben Disziplin oder aus Nachbardisziplinen sind. Die übergeordnete Fragestellung unserer Studie will klären, wie stark die oben beschriebenen strukturellen Einflüsse auf die Kommunikationswissenschaft deren Normativität prägen: 


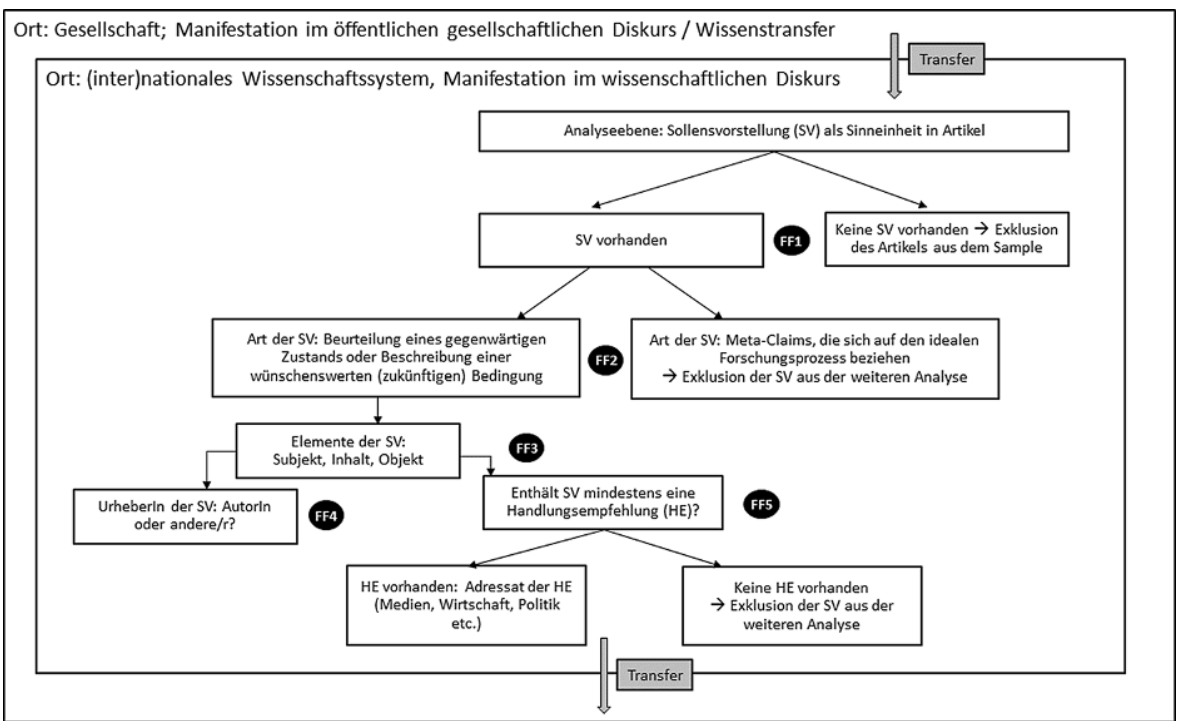

Abb. 1 Manifestationen von Normativität in Fachzeitschriftenaufsätzen

\section{Unterscheidet sich die Normativität von Fachzeitschriftenaufsätzen in Deutsch- land und den USA?}

Die erste Manifestationsstufe von Normativität sind Sollensvorstellungen, mit denen Forschende normative Vorstellungen - bewusst oder unbewusst - zum Ausdruck bringen. Je mehr Sollensvorstellungen eine Publikation enthält, desto normativer ist sie nach diesem Verständnis. Wir berücksichtigen nur Sollensvorstellungen, die sich auf gesellschaftliche Zustände oder Entwicklungen beziehen. Forschungsbezogene Sollensvorstellungen (z.B. Forderungen nach der ,Replikation mit einer größeren Stichprobe“) fließen nicht in die weitere Analyse ein.

FF1 Wie viele Sollensvorstellungen werden in den Aufsätzen durchschnittlich genannt?

Im nächsten Schritt erfassen wir, ob eine Sollensvorstellung einen gegenwärtigen Zustand bewertet oder einen wünschenswerten zukünftigen Zustand beschreibt. Letzteres interpretieren wir als stärkere Manifestation von Normativität, weil der Anspruch, etwas zu erreichen bzw. einen Wandel herbeizuführen, voraussetzungsvoller ist und mehr Aktivität der Verantwortlichen erfordert als die Bewertung eines bereits bestehenden Zustandes bzw. dessen Beibehaltung.

FF2 Wie häufig beschreiben Sollensvorstellungen wünschenswerte gegenwärtige, wie häufig zukünftige Zustände?

Um herauszufinden, worauf sich die geäußerten normativen Vorstellungen beziehen, analysieren wir die drei Elemente einer Sollensvorstellung: Inhalt, Subjekt und Objekt. Hieraus lässt sich z. B. ablesen, zu welchen gesellschaftlichen Phänomenen sich die Autorinnen normativ äußern und wen sie in der Verantwortung sehen. 
FF3 Welche Inhalte, Subjekte und Objekte von Sollensvorstellungen werden angesprochen?

Anschließend untersuchen wir, ob die Autorinnen selbst Sollensvorstellungen formulieren oder lediglich Sollensvorstellung aus anderen Quellen (z. B. Personen, Institutionen) wiedergeben. Ersteres sehen wir aufgrund der Selbstpositionierung als stärkere Manifestation von Normativität an.

FF4 Wer sind die Urheberinnen der Sollensvorstellungen?

Am stärksten manifestiert sich Normativität, wenn eine Sollensvorstellung mit einer Handlungsempfehlung für Adressatinnen verknüpft wird, weil dann ein Transfer in den öffentlichen Diskurs angestrebt wird. Das kann ein wichtiger Schritt hin zur Entwicklung ,,von Kommunikations- und Kooperationsformen zwischen Wissenschaft und Praxis“ sein, ,die zu einem Abbau der gewachsenen Kommunikationsbarrieren und zu einer Neubestimmung des Verhältnisses von sozialer Distanz und Anschlussfähigkeit an die gesellschaftliche Praxis“ (Howaldt 2005, S. 192) beitragen.

FF5 Wie häufig werden Handlungsempfehlungen ausgesprochen und an wen richten sie sich?

Offen bleibt in der vorliegenden Studie, inwieweit Handlungsempfehlungen angenommen und umgesetzt werden.

\section{Methode}

\subsection{Sample}

Der Beantwortung dieser Forschungsfragen dient eine quantitative Inhaltsanalyse kommunikationswissenschaftlicher Fachzeitschriftenaufsätze, die zwischen 1970 und 2014 publiziert wurden. Der Untersuchungszeitraum umfasst somit eine Zeit älterer und jüngerer intensiver Umbrüche in Gesellschaft, Wissenschaft und Mediensystemen (z. B. Wertewandel, Dualisierung der europäischen Rundfunksysteme, Digitalisierung), welche die Arbeitsbedingungen und Forschungsgegenstände von Kommunikationswissenschaftlerinnen herausfordern und folglich normative Äußerungen hervorrufen dürften. Fachzeitschriftenaufsätze bilden die Kommunikationswissenschaft nicht in ihrer Gesamtheit ab. Sie gelten aktuell aber als sichtbarste Publikationsform mit der höchsten Reputation und geben Hinweise auf zentrale Problemstellungen, mit denen sich das Fach beschäftigt.

Das Fachzeitschriftensample (Tabelle A1 im zusätzlichen Anhang der Online-Version dieses Aufsatzes) enthält die drei wichtigsten deutschsprachigen Fachzeitschriften (Publizistik, Medien \& Kommunikationswissenschaft (M\&K; vormals Rundfunk und Fernsehen), Studies in Communication and Media (SCM)) sowie 20 internationale englischsprachige Fachzeitschriften. Letztere identifizierten wir folgendermaßen im Web of Science: Aus den 74 dort als „kommunikationswissenschaftlich“ aufgeführten Fachzeitschriften wurden nicht-englischsprachige Zeitschriften sowie Zeitschriften aus benachbarten Disziplinen wie Wirtschaftswissenschaften oder Lin- 
guistik als irrelevant ausgeschlossen. Von den verbleibenden 43 Zeitschriften gingen die 20 mit dem höchsten Impact Factor (ohne Eigenzitate) ins Sample ein.

Ausgehend von Pretests legten wir eine Zielgröße von ca. 3000 Sollensvorstellungen fest, um trotz der zu erwartenden Diversität hinreichende Fallzahlen für detaillierte Analysen sicherzustellen. Um dies zu erreichen, wählten wir aus allen 346 Heften der deutschsprachigen Zeitschriften, die im Untersuchungszeitraum veröffentlicht wurden, 80 Hefte $(23 \%)$ und in diesen wiederum jeweils einen Aufsatz zufällig aus. Aus allen 2616 Heften der internationalen Zeitschriften, die im Untersuchungszeitraum veröffentlicht wurden, zogen wir zufällig 400 Hefte (15\%) und in diesen wiederum jeweils einen Aufsatz. Buchbesprechungen, Berichte und Nachrufe schlossen wir aus. Die unterschiedlichen Quoten (23 vs. 15\%) tragen der deutlich geringeren Gesamtzahl der deutschsprachigen Hefte Rechnung und sorgen im deutschsprachigen Sample für eine hinreichende Zahl an Sollensvorstellungen. Insgesamt identifizierten wir auf diese Weise 5496 Sollensvorstellungen.

Da unser Forschungsinteresse dem Vergleich deutscher und US-amerikanischer Fachzeitschriftenaufsätze gilt, gingen in die Auswertung nur Aufsätze mit mindestens einer Autorin mit deutscher Affiliation aus den drei deutschen Fachzeitschriften (deutsches Sample) sowie Aufsätze mit mindestens einer Autorin mit US-amerikanischer Affiliation aus den 20 internationalen Fachzeitschriften (US-Sample) ein. ${ }^{3}$ 157 Aufsätze, auf die dieses Kriterium nicht zutrifft, und die darin enthaltenen 2263 Sollensvorstellungen wurden aus der Analyse ausgeschlossen. Hieraus ergibt sich eine Stichprobe von 326 Aufsätzen $\left(n_{d t}=61, n_{u s}=265\right)$, in denen anhand des nachfolgend beschriebenen Verfahrens 3233 Sollensvorstellungen $\left(n_{d t}=691, n_{u s}=2542\right)$ identifiziert wurden.

\subsection{Codierung von Sollensvorstellungen}

Sollensvorstellungen in Fachzeitschriftenaufsätzen standardisiert zu codieren, ist eine komplexe Aufgabe, die ein hohes Maß an theoretischer und semantischer Sensibilität von den Codiererinnen verlangt: Weil Forschende normative Äußerungen nur selten explizit als solche bezeichnen (vgl. Althaus 2012, S. 99), eignet sich eine Stichwortsuche nach Sollensvorstellungen nicht (z. B. anhand von Suchtermini wie „wert*“, „norm*“, die dann auch zu Treffern wie „,der Wert des Geldes“ führen). Zudem ist die Vielfalt der Normen und Werte, auf die ein interdisziplinäres

\footnotetext{
3 Welche Aufsätze von US-amerikanischen bzw. deutschen Autorinnen stammen, lässt sich anhand unterschiedlicher Kriterien bestimmen. Im US-Sample unterscheidet sich die Fallzahl der relevanten Aufsätze kaum, egal welches Kriterium herangezogen wird (alle Autorinnen aus den USA: n= 243 Aufsätze; Erstautorin aus den USA: $n=254$ Aufsätze; mindestens $50 \%$ aller Autorinnen aus den USA: $n=262$ Aufsätze; mindestens eine Autorin aus den USA: $n=265$ Aufsätze). Offenbar publizieren US-Autorinnen weit überwiegend zusammen mit anderen Autorinnen aus den USA. Das konservativste Kriterium - sämtliche Autorinnen kommen aus dem jeweiligen Land - führt im erheblich kleineren deutschsprachigen Sample zu einer starken Reduktion der Fallzahlen ( $n=56$ Aufsätze) und würde die Belastbarkeit der Befunde einschränken. Deshalb haben wir uns für das beschriebene weichere Kriterium - mindestens eine Autorin kommt aus Deutschland bzw. aus den USA - entschieden. Die Ko-Autorinnen der deutschen Aufsätze kommen mehrheitlich aus Österreich und/oder der Schweiz. Zwischen diesen Communities besteht ein enger wissenschaftlicher und personeller Austausch, und auch die Wissenschafts- und Mediensysteme sind ähnlich.
} 

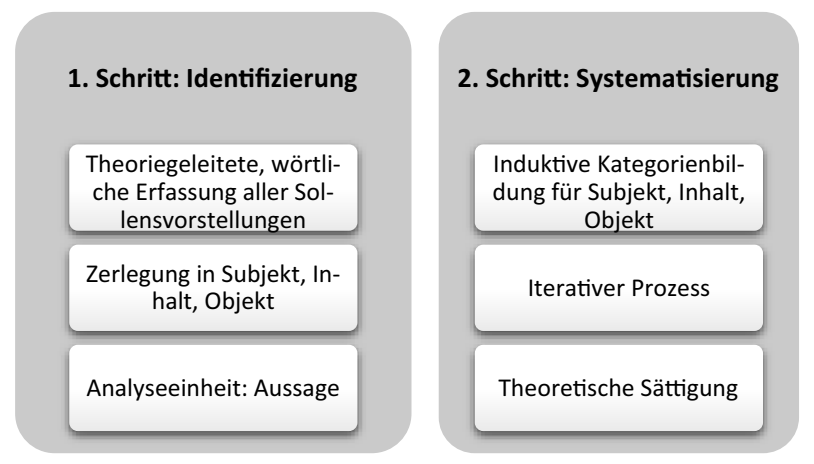

\section{Schritt: Kategorisierung}

Standardisierte Codierung von Subjekt, Inhalt, Objekt

Subjekt/Objekt: 9 Oberund 75 Unterkategorien

Inhalt: 8 Ober- und 65 Unterkategorien

Abb. 2 Mehrstufige Codierung von Sollensvorstellungen

Fach wie die Kommunikationswissenschaft referiert, kaum überschaubar, interkulturell verschieden und möglicherweise einem zeitlichen Wandel unterworfen. Die Vorabdefinition bestimmter Normen und Werte in einem Codeplan würde demnach zwangsläufig zu blinden Flecken führen: Es ließen sich nur diejenigen finden, nach denen gesucht wurde. Daher wendeten wir in dieser Studie ein dreistufiges Vorgehen zur Identifikation, Systematisierung und Kategorisierung von Sollensvorstellungen an (Abb. 2), das sich an die von Früh (2001, S. 72-74) beschriebene Kombination aus theorie- und empiriegeleiteter Kategorienbildung anlehnt. Innovativ daran ist, dass wir diese mit dem Konzept der Sollensvorstellungen von Esser (2000) verknüpfen. Wir schlagen damit erstmals ein methodisches Verfahren vor, mit dem sich die Normativität des Untersuchungsmaterials messen lässt, ohne die zu erfassenden Werte und Normen vorab in Listen festzuschreiben und damit den Erkenntnisgewinn einzuschränken.

Schritt 1: Identifizierung Im ersten Schritt identifizierten wir alle Sollensvorstellungen $(n=5496)$ theoriegeleitet entsprechend der genannten Definition.

Um die bestmögliche Reliabilität sicherzustellen, beschränkten wir uns auf explizit formulierte (manifeste) Sollensvorstellungen und schlossen implizite Sollensvorstellungen aus (z.B. konditionale Formen wie „könnte“, Stilmittel wie Ironie). Signalwörter wie „,sollen“, ,,erfordern“, ,wichtig“ oder „,negativ“ sind ein Indiz für Sollensvorstellungen, mussten aber nicht zwingend vorhanden sein, damit eine Aussage als Sollensvorstellung kodiert wurde. Wir erfassten sowohl die Sollensvorstellung in ihrer Gesamtheit als auch ihre drei Elemente Inhalt, Subjekt und Objekt in ihrer Originalformulierung als Freitext. Zudem erhoben wir standardisiert Art und Urheber der Sollensvorstellungen sowie - falls vorhanden - Handlungsempfehlungen und deren Adressatinnen.

Schritt 2: Systematisierung Im zweiten Schritt systematisierten wir die erfassten Inhalte, Subjekte und Objekte der Sollensvorstellungen und verdichteten sie induktiv zu theoretisch gesättigten Kategorien für die quantitative Codierung. Für die Subjekte und Objekte bezogen wir hierbei alle Codierungen aus Schritt 1 ein. Auf Basis dieser Systematisierung und mehrerer Pretests erstellten wir empiriegeleitet einen 
Schlüsselplan für Subjekte und Objekte mit neun gesellschaftlichen Bereichen als Obercodes (z. B. Mediensystem) und 75 Untercodes (z. B. Journalistinnen, Medienregulierung; siehe zusätzlicher Anhang der Online-Version dieses Aufsatzes). Für die Inhalte zogen wir eine Zufallsstichprobe von 12\% aller in Schritt 1 erfassten Sollensvorstellungen $(n=646)$, die von zwei Forscherinnen qualitativ ausgewertet wurden. Auf Basis dieses iterativen Prozesses sowie anschließender Pretests entwickelten wir empiriegeleitet einen Schlüsselplan mit acht gesellschaftlichen Bereichen als Obercodes (z.B. Funktionen und Gütekriterien von Journalismus und Massemedien) mit 65 Untercodes (z. B. Informationen bereitstellen und vermitteln, Kontrolle gesellschaftlicher und politischer Eliten; siehe zusätzlicher Anhang der Online-Version dieses Aufsatzes).

Schritt 3: Kategorisierung Entsprechend dieser Schlüsselpläne codierten zwei studentische Codiererinnen alle im Freitext erfassten Inhalte, Subjekte und Objekte der Sollensvorstellungen in einer standardisierten Inhaltsanalyse.

\subsection{Variablen}

Inhalt der Sollensvorstellung Für jede Sollensvorstellung codierten wir, was ihr zufolge zu verantworten ist. Die darin enthaltene Erwartung konnte positiv (was soll geschehen/getan werden?) oder negativ (was soll verhindert/vermieden werden?) formuliert sein. Der Inhalt ist das Kernstück der Sollensvorstellung. Nur wenn er vorhanden ist, liegt eine Sollensvorstellung vor.

Subjekt der Sollensvorstellung Das Subjekt der Sollensvorstellung ist theoretisch definiert als Adressatin, die für die Umsetzung des Inhalts verantwortlich ist. Allerdings führte diese Definition in mehreren Pretests zu einer geringen Reliabilität. Da Adressatinnen sehr oft mit dem grammatikalischen Subjekt des Satzes identisch sind (z.B. „Der Presserat muss Verstöße gegen den Pressekodex aktiver ahnden“ „der Presserat“ ist zugleich grammatikalisches Subjekt und adressierte Institution der Sollensvorstellung), legten wir das grammatikalische Subjekt operational als Subjekt der Sollensvorstellung fest. Das Subjekt kann eine individuelle Akteurin (z. B. eine Politikerin, ein Elternteil), eine kollektive Akteurin (z.B. eine Gruppe von Personen, eine Institution), eine Sache (z. B. gesundheitsbezogene Information) oder eine Entwicklung (z.B. die Kommerzialisierung des Rundfunksystems) sein. Anders als der Inhalt muss ein Subjekt nicht zwingend vorhanden sein. Eine Sollensvorstellung kann ein oder auch mehrere Subjekte umfassen. Das empirische Maximum lag bei sieben Subjekten in einer Sollensvorstellung.

Objekt der Sollensvorstellung Das Objekt der Sollensvorstellung gibt an, wer von der Realisierung ihres Inhalts betroffen ist. Ebenso wie das Subjekt kann das Objekt der Sollensvorstellung eine individuelle oder kollektive Akteurin, eine Sache oder eine Entwicklung sein. Auch ein Objekt muss nicht zwingend vorhanden sein. Eine Sollensvorstellung kann ein oder auch mehrere Objekte enthalten. Das empirische Maximum lag bei fünf Objekten in einer Sollensvorstellung. 
Art der Sollensvorstellung Für jede Sollensvorstellung erhoben wir, ob darin ein gegenwärtiger Zustand bewertet oder ein erstrebenswerter zukünftiger Zustand beschrieben wurde.

Urheberin der Sollensvorstellung Für jede Sollensvorstellung erfassten wir, ob sie von den Autorinnen des Aufsatzes selbst geäußert wurde oder ob andere Quellen (z.B. andere Forschende, Pressekodex, Grundgesetz) als Urheberinnen genannt wurden.

Handlungsempfehlungen Für jede Sollensvorstellung codierten wir, ob die Autorinnen daraus Handlungsempfehlungen ableiten. Diese wurden operationalisiert als konkrete Anleitungen oder Ratschläge, wie bestimmte Adressatinnen künftig handeln sollten, um einen wünschenswerten Zustand herzustellen. Sie richten sich an eine klar erkennbare, eindeutig benannte Adressatin(nengruppe). Somit beinhaltet nicht jede Sollensvorstellung, für die ein Subjekt codiert wurde, automatisch eine Handlungsempfehlung.

Adressatin der Handlungsempfehlung Für jede Sollensvorstellung, die eine Handlungsempfehlung enthielt, erfassten wir bis zu drei individuelle oder kollektive Adressatinnen. Codiert wurden hier Referenzen auf die Medienpraxis, die Wissenschaft, die Gesellschaft, die Politik/Gesetzgebung, die Wirtschaft/Industrie, individuelle Akteurinnen und sonstige Adressatinnen.

Forschungsbereich Zur Kontextualisierung der Ergebnisse wurde für jeden Artikel erfasst, in welchen Forschungsbereichen entsprechend der Lasswell-Formel (vgl. Lasswell 1948) er verortet ist: Kommunikatorforschung, Medienforschung, Inhaltsforschung sowie Rezeptions-/Wirkungsforschung. ${ }^{4}$ Ein Artikel und die in ihm enthaltenen Sollensvorstellungen konnten zwischen einem und vier Forschungsbereichen zugeordnet werden.

\subsection{Reliabilität}

Der Intercoder-Reliabilitätstest (Krippendorff's $\alpha$ ) erbrachte fast durchweg gute Werte (Identifikation von Sollensvorstellungen (SV) inkl. true negatives: 0,67; Subjekt der SV: 0,97; Objekt der SV: 0,88; Art der SV: 0,89; Urheberin der SV: 0,99; Adressatinnen der Handlungsempfehlung (HE) 1-3: 0,80/0,71/0,89). Lediglich für den Inhalt der Sollensvorstellung $(0,60)$ wäre ein höherer Koeffizient wünschenswert. Dennoch weist dieser Koeffizient auf eine deutlich überzufällige Übereinstimmung zwischen den Codiererinnen hin. Zu bedenken ist die Vielschichtigkeit und Komplexität der Phänomene, die mit dieser Variable erfasst werden. Weil für explorative, komplexe, anspruchsvolle Codierungen generell geringere Koeffizienten zu erwarten sind (vgl. Feng 2013) und es keinen „fixed numerical cut-off point“ (Krippendorff 2008, S. 5) gibt, beziehen wir diese Variable in die Analyse ein.

\footnotetext{
4 Aufgrund der mangelnden Trennschärfe zwischen Rezeptions- und Wirkungsforschung wurden diese beiden Forschungsbereiche zusammengefasst.
} 
Tab. 1 Durchschnittliche Anzahl an Sollensvorstellungen (SV) in deutschen und US-amerikanischen Fachzeitschriftenaufsätzen

\begin{tabular}{llll}
\hline & $\begin{array}{l}\text { Deutschland } \\
n=61 \text { Aufsätze }\end{array}$ & USA & Gesamt \\
& 11,33 & 9,59 & $n=326$ Aufsätze \\
\hline M (SV) & 12,184 & 12,223 & 9,92 \\
SD (SV) & 7,0 & 6,0 & 12,216 \\
Md (SV) & & 6,5 \\
\hline
\end{tabular}

$\mathrm{t}(324)=-1,000 ; \mathrm{n} . \mathrm{s}$.

\section{Ergebnisse}

\subsection{Häufigkeit (FF1) und Art der Sollensvorstellungen (FF2)}

Der Grad der Normativität der Fachzeitschriftenaufsätze wird anhand der durchschnittlichen Anzahl an Sollensvorstellungen pro Aufsatz gemessen (FF1). In der großen Mehrheit der deutschen wie US-amerikanischen Aufsätze ist mindestens eine Sollensvorstellung enthalten (Deutschland: $90 \%$; USA: $88 \% ; \chi^{2}(1, N=326)=0,322$; n. s.). Auch die durchschnittliche Anzahl an Sollensvorstellungen pro Aufsatz - 11,3 in Deutschland, 9,6 in den USA - unterscheidet sich zwischen den beiden Samples nicht signifikant (Tab. 1). Gemessen an diesem Indikator ist die Kommunikationswissenschaft in beiden Ländern gleich normativ.

Für alle weiteren Auswertungen wechselt die Perspektive von der Artikelebene zur Ebene der Sollensvorstellungen, beginnend mit der Art der Sollensvorstellung (FF2). In der deutlichen Mehrheit der Fälle beschreiben Sollensvorstellungen einen erstrebenswerten zukünftigen Zustand; wesentlich seltener bewerten sie einen gegenwärtigen Zustand (69 vs. $31 \%$ ). In Deutschland (81 vs. 19\%) ist diese Tendenz signifikant stärker ausgeprägt als in den USA (65 vs. $35 \%)\left(\chi^{2}(1, N=3233)=65,158\right.$; $p<0,001$; Cramer's V=0,142).

\subsection{Inhalt, Subjekt und Objekt der Sollensvorstellungen (FF3)}

\subsubsection{Inhalt der Sollensvorstellung}

Im Folgenden geht es um die drei Elemente der Sollensvorstellungen $(F F 3)$, beginnend mit ihrem Inhalt als zentralem Element. Wir vergleichen zunächst den Stellenwert der fünf häufigsten Inhaltsbereiche allgemein und betrachten dann die Unterschiede zwischen den Samples innerhalb dieser Bereiche und zwischen zentralen Forschungsbereichen detaillierter.

Abb. 3 zeigt, dass sich die Sollensvorstellungen sowohl in Deutschland (31\%) als auch in den USA (32\%) am häufigsten auf Funktionen bzw. Gütekriterien von Wissenschaftlerinnen/Wissenschaft (z.B. Verstehen und Erklären des Analysegenstandes) beziehen. Kommunikationswissenschaftliche Fachzeitschriftenaufsätze sind also bemerkenswert selbstreferenziell - obwohl wir Sollensvorstellungen, die sich unmittelbar auf den Forschungsprozess beziehen, ausgeschlossen haben (also z. B. Erwartungen bezüglich einer wünschenswerten Stichprobengröße). Hinsichtlich der weiteren Inhalte unterscheiden sich beide Samples dagegen signifikant: Funktionen/ 
$60 \%$

$50 \%$

$40 \%$

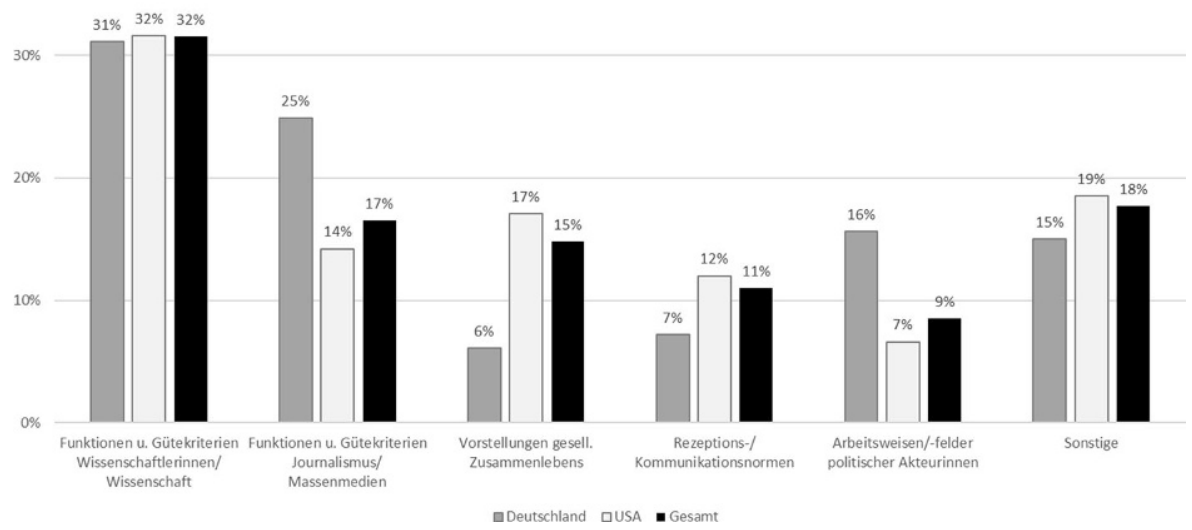

Abb. 3 Inhalte der Sollensvorstellungen (SV; in \%) (Basis: $n=3229$ SV ( $\left.\mathrm{n}_{\mathrm{dt}}=691 \mathrm{SV} ; \mathrm{n}_{\mathrm{US}}=2538 \mathrm{SV}\right) \cdot \chi^{2}$ $(8, N=3229)=169,665 ; p<0,001$; Cramer's $\mathrm{V}=0,229$. Inhalte, die insgesamt weniger als $7 \%$ aller Inhalte ausmachen, werden unter „Sonstige“ zusammengefasst)

Gütekriterien von Journalismus/Massenmedien (z. B. Qualitätssicherung in Medienbetrieben) werden in Deutschland am zweithäufigsten thematisiert (25\%) - erheblich häufiger als in den USA (14\%, Rang 3). Dort liegen auf Rang 2 die Vorstellungen eines gelungenen gesellschaftlichen Zusammenlebens (z. B. physische und psychische Gesundheit; $17 \%$ ), deren Anteil dreimal so groß ist wie im deutschen Sample (6\%, Rang 5). Bedeutsamer in den USA (12\%, Rang 4) sind auch Rezeptions- und Kommunikationsnormen (z.B. Medienkompetenz; Deutschland: $7 \%$, Rang 4). Im deutschsprachigen Sample sind dagegen Arbeitsweisen und -felder politischer Akteurinnen stärker vertreten (z. B. Politikvermittlung, Ethik der Politik; Deutschland: $16 \%$, Rang 3; USA: $7 \%$, Rang 5).

Auch innerhalb dieser fünf Bereiche unterscheidet sich die Wichtigkeit bestimmter normativer Vorstellungen signifikant zwischen beiden Samples (siehe Tabelle A2 im zusätzlichen Anhang der Online-Version dieses Aufsatzes). Die Gütekriterien von Wissenschaft(lerinnen) thematisieren zwar in beiden Samples in gut jeder zweiten Sollensvorstellung das Verstehen und Erklären, daneben gibt es aber einige deutliche Unterschiede. Der größte besteht darin, dass US-Autorinnen fast dreimal so häufig die Wichtigkeit empirischen Forschens betonen (plus 14 Prozentpunkte [PP] gegenüber dem deutschen Sample). Die deutschen Autorinnen hingegen referieren häufiger auf Inter- und Transdisziplinarität (plus 9 PP gegenüber dem US-amerikanischen Sample) sowie den Realitätsbezug der Forschung (plus 9 PP). 
Die Autorinnen nennen sehr vielfältige Funktionen/Gütekriterien von Journalismus/Massenmedien, jedoch mit unterschiedlichen Prioritäten: US-amerikanische Autorinnen heben häufiger hervor, dass die Medien Aufmerksamkeit wecken (plus 4 PP) und Vielfalt gewährleisten sollten (plus 4 PP). Deutsche Autorinnen betonen hingegen öfter Objektivität, Neutralität und Verifizierbarkeit (plus 8 PP) sowie die Wichtigkeit der Qualitätssicherung in den Medien (plus 4 PP).

Auch die Vorstellungen eines gelungenen gesellschaftlichen Zusammenlebens differieren erheblich zwischen beiden Samples: US-Autorinnen nehmen hierauf deutlich häufiger ganz allgemein Bezug, d.h. ohne konkrete Normen und Werte zu benennen (plus 15 PP). Thematisieren sie konkrete Werte, legen sie besonderen Wert auf ,healthy living“, psychische und physische Gesundheit (plus 19 PP) ein Aspekt, der im deutschen Sample nur in einer einzigen Sollensvorstellung vorkommt. In Deutschland werden dagegen stärker Bildung, Informiertheit und Wissen (plus 13 PP), Partizipation/Mitbestimmung (plus 8 PP) und soziale Integration/ Inklusion (plus $8 \mathrm{PP}$ ) betont.

Die Sollensvorstellungen zu Rezeptions- und Kommunikationsnormen machen besonders deutlich, welche großen inhaltlichen Unterschiede sich im Detail hinter ähnlichen Ergebnissen verbergen können: Die US-amerikanischen Aufsätze thematisieren viel häufiger Konversationsnormen (z.B. Etikette von interpersonaler Kommunikation; plus 27 PP), die deutschen erheblich öfter Normen der Mediennutzung und Informationsverarbeitung (z. B. Informationsaufnahme, Medienkompetenz; plus $18 \mathrm{PP}$ ).

Innerhalb der Arbeitsweisen und-felder politischer Akteurinnen entfallen mehr als die Hälfte aller Sollensvorstellungen (insgesamt 52\%) auf Medienpolitik, Medienregulierung und Media Governance. In Deutschland ist dieses Thema erheblich zentraler als in den USA (plus 36 PP). Alle anderen Inhalte in diesem Bereich kommen nur selten vor; die entsprechenden Unterschiede zwischen den beiden Samples sind daher nicht belastbar.

Die übergreifende Betrachtung eines so breiten Fachs wie der Kommunikationswissenschaft kann Unterschiede zwischen verschiedenen Forschungsfeldern verdecken. Daher vergleichen wir im nächsten Schritt, wie stark deutsche und USamerikanische Aufsätze die fünf wichtigsten Inhalte der Sollensvorstellungen in den vier zentralen Forschungsbereichen der Kommunikationswissenschaft ansprechen. Tab. 2 zeigt, dass deutsche Aufsätze auch in jedem der vier Forschungsfelder Funktionen und Gütekriterien von Wissenschaft(lerinnen) thematisieren. Die stärkere Selbstreferenzialität der deutschen Kommunikationswissenschaft ist offenbar ein übergreifendes Phänomen. US-amerikanische Aufsätze thematisieren auch in einzelnen Forschungsbereichen häufiger Vorstellungen eines gelungenen gesellschaftlichen Zusammenlebens. Auffällig ist auch, dass in der Kommunikatorforschung vier von fünf zentrale Inhalte prozentual häufiger in den USA thematisiert werden. In der Rezeptions- und Wirkungsforschung hingegen kommen vier von fünf zentrale Inhalte prozentual öfter in Deutschland vor. Bei der Interpretation dieser Befunde ist aber zu beachten, dass viele Sollensvorstellungen mehreren Forschungsbereichen zugeordnet sind, weshalb die Befunde nur bedingt auf die Spezifika des jeweiligen Forschungsfeldes zurückgeführt werden können. 
Wie normativ ist die Kommunikationswissenschaft?

109

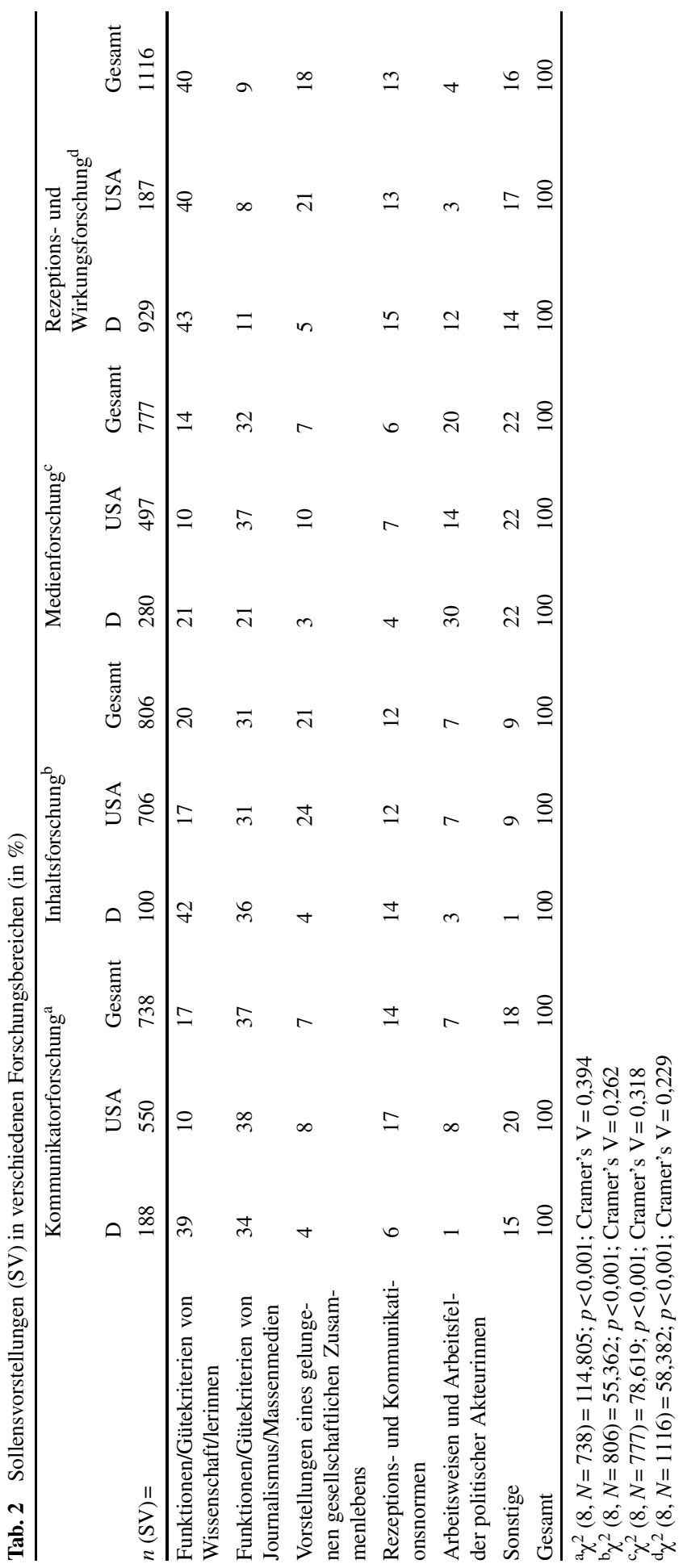

Springer 


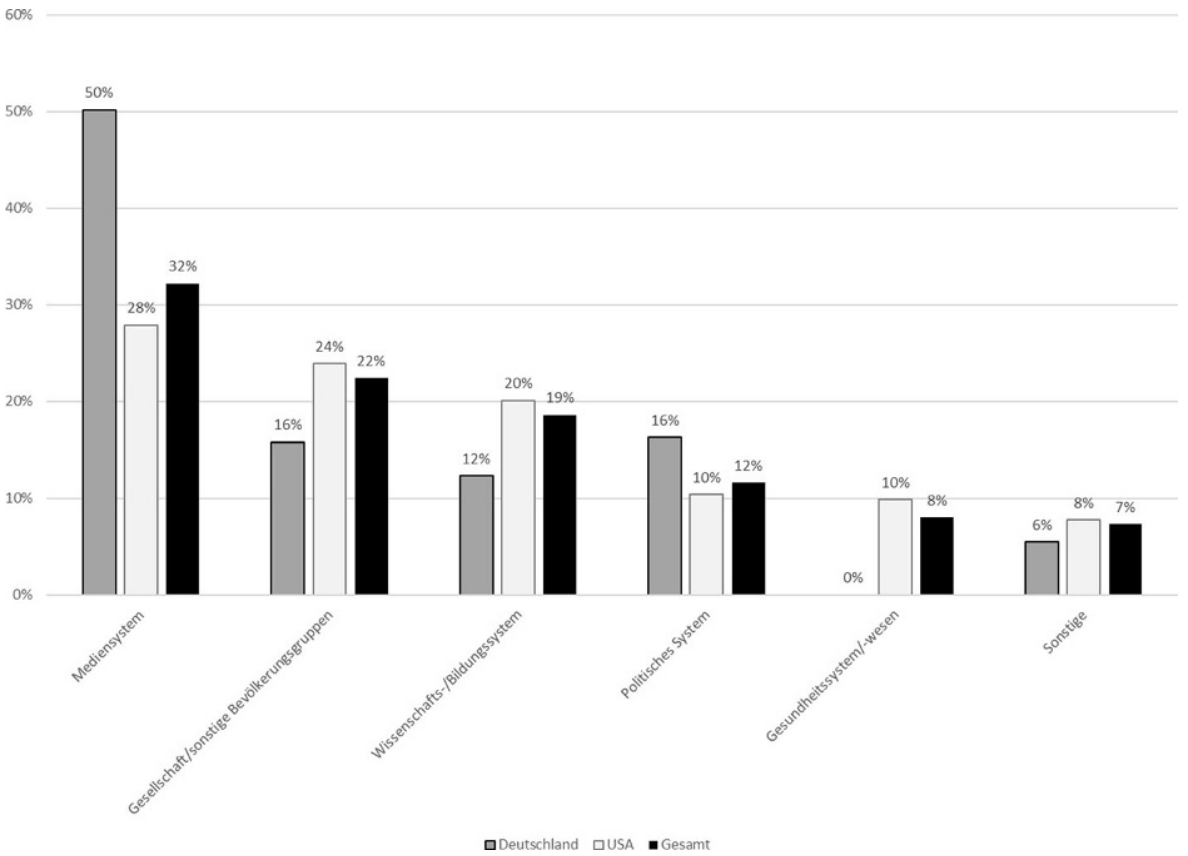

Abb. 4 Subjekte der Sollensvorstellungen (SV; in \%) (Basis: $n=1055$ Subjekte $\left(\mathrm{n}_{\mathrm{dt}}=203\right.$ Subjekte; nus $=852$ Subjekte). $\chi^{2}(9, N=1055)=72,446 ; p<0,001$; Cramer's V=0,262. Subjekte, die insgesamt weniger als $7 \%$ aller Subjekte ausmachen, werden unter ,Sonstige“ zusammengefasst)

\subsubsection{Subjekt der Sollensvorstellung}

Nur in rund jeder dritten Sollensvorstellung (31\%; Deutschland: $27 \%$; USA: $32 \%$; $\chi^{2}(1, N=3229)=7,022 ; p=0,005$; Cramer's V = 0,049) wird mindestens ein Subjekt adressiert. Die Autorinnen bewerten also häufig aktuelle oder formulieren erstrebenswerte Zustände, geben aber nicht an, wer dafür verantwortlich sein soll. Die Subjekte unterscheiden sich zwischen den Ländern signifikant und bestätigen den stärkeren Bezug normativer Äußerungen auf Journalismus und Massenmedien in den deutschen Aufsätzen bzw. auf die Gesellschaft in den US-Aufsätzen (Abb. 4): Häufigstes Subjekt der Sollensvorstellungen ist insgesamt das Mediensystem; im deutschen Sample (50\% der Subjekte) kommt es aber fast doppelt so häufig vor wie im US-Sample (28\%). Sollensvorstellungen der deutschen Artikel beziehen sich rund dreimal so oft auf das Mediensystem wie auf die Gesellschaft und einzelne Bevölkerungsgruppen (z. B. Familien, Touristinnen, Inhaftierte; 16\%). Im USSample hingegen nehmen gesellschaftliche Bezugsgruppen mit 24\% - fast gleich bedeutsam wie das Mediensystem - Rang zwei der Subjekte ein. Subjekte aus dem Wissenschafts- und Bildungssystem sind im US-Sample (20\%; Rang 3) zentraler als im deutschen (12\%, Rang 4), Subjekte aus dem politischen System hingegen sind im deutschen Sample (16\%, Rang 3) bedeutsamer (USA: 10\%, Rang 4). Auffällig ist, dass Akteurinnen aus dem Gesundheitssystem im US-Sample $10 \%$ aller 
$60 \%$

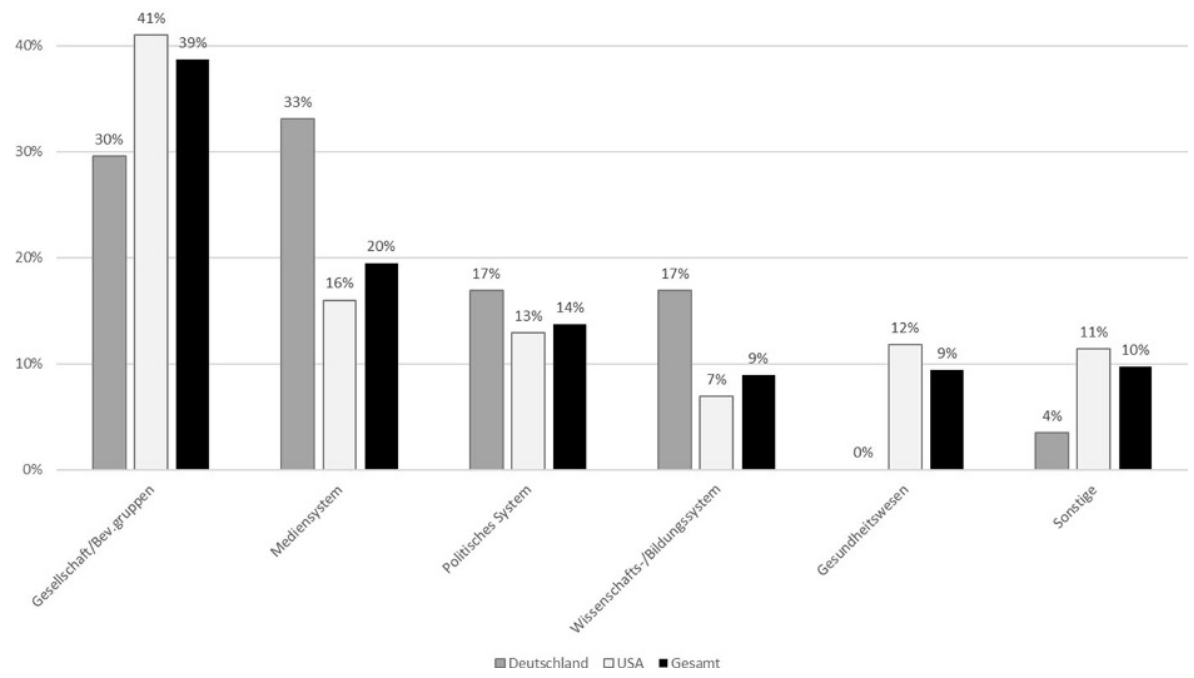

Abb. 5 Objekte der Sollensvorstellungen (SV; in \%) (Basis: $n=693$ Objekte $\left(\mathrm{n}_{\mathrm{dt}}=142\right.$ Objekte; $\mathrm{n}_{\mathrm{US}}=551$ Objekte). $\chi^{2}(9, N=693)=61,816 ; p<0,001$; Cramer's V $=0,299$. Objekte, die insgesamt weniger als $7 \%$ aller Objekte ausmachen, werden unter „Sonstige“ zusammengefasst)

Subjekte ausmachen (Rang 5). Im deutschen Sample kommen sie hingegen nicht vor.

\subsubsection{Objekt der Sollensvorstellung}

Objekte der Sollensvorstellungen, d. h. die von ihnen Betroffenen, werden noch seltener genannt als die Subjekte, nämlich nur in jeder fünften Sollensvorstellung $(21 \%$; Deutschland: 20\%; USA: $21 \% ; \chi^{2}(1, N=3233)=0,615$; n. s.; Cramer's V=0,014). Obwohl die Gesellschaft und bestimmte Bevölkerungsgruppen in beiden Samples zentrale Objekte sind (Abb. 5), fällt ihre signifikant größere Bedeutung in den USA (41\% der Objekte; Rang 1) ins Auge (Deutschland: 30\%, Rang 2). Das Mediensystem hingegen ist im deutschen Sample (33\%; Rang 1) doppelt so häufig Objekt einer Sollensvorstellung (USA: 16\%; Rang 2). Objekte aus dem politischen System werden in beiden Ländern ähnlich häufig angesprochen (Deutschland: 13\%, Rang 3; USA: $17 \%$, Rang 3). Auf das Wissenschafts- und Bildungssystem wird in Deutschland (17\%; Rang 3) häufiger Bezug genommen, jedoch erscheint es nicht zentral (USA: 7\%, Rang 5). Das Gesundheitssystem kommt als Objekt nur in den USA vor (11\%, Rang 4; Deutschland: 0\%). 
$60 \%$

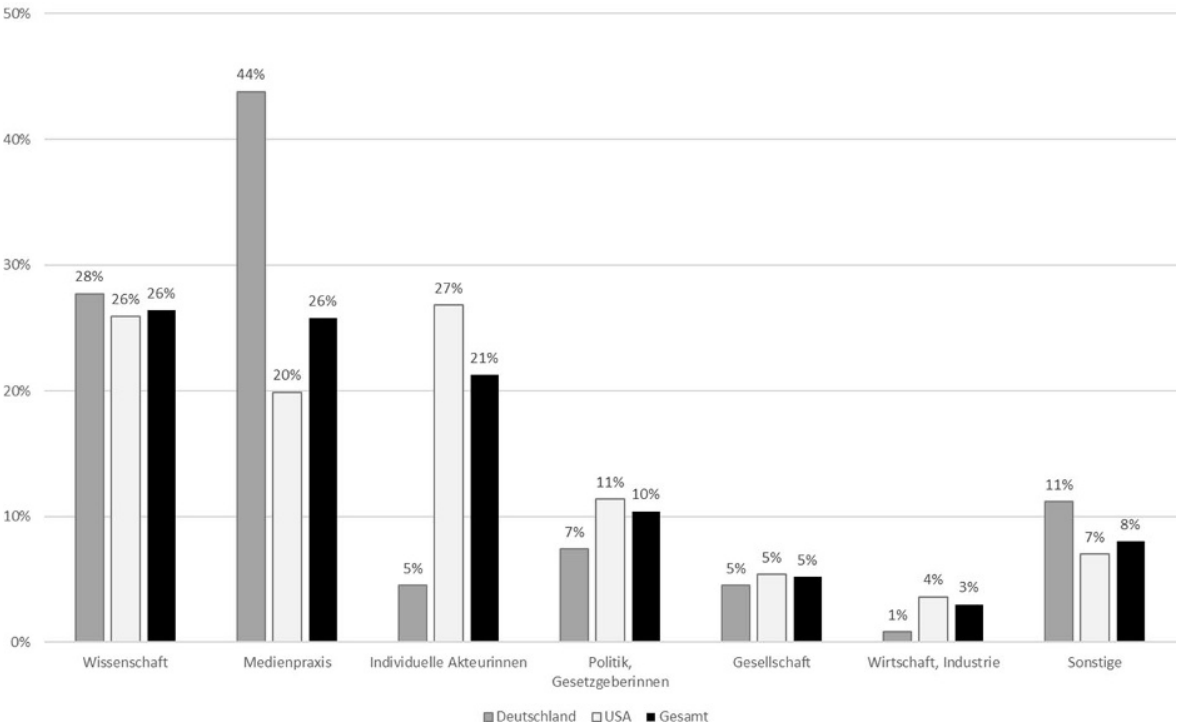

Abb. 6 Adressatinnen der Handlungsempfehlungen (in \%) (Basis: $n=982$ Adressatinnen $\left(\mathrm{n}_{\mathrm{dt}}=242\right.$ Adressatinnen; nus $=742$ Adressatinnen). $\chi^{2}(6, N=982)=94,771 ; p<0,001$; Cramer's V=0,311)

\subsection{Urheberinnen der Sollensvorstellungen (FF4)}

Urheberinnen der Sollensvorstellungen $(F F 4)$ sind in beiden Ländern vor allem die Autorinnen der Fachzeitschriftenaufsätze selbst. Deutlich seltener zitieren sie normative Positionen anderer Urheberinnen (72 vs. 28\%). Deutsche Autorinnen (78 vs. $23 \%$ ) äußern signifikant häufiger ihre eigenen normativen Positionen als US-amerikanische Autorinnen (70 vs. 30\%) $\left(\chi^{2}(1, N=3233)=18,997 ; p<0,001\right.$; Cramer's V=0,077).

\subsection{Handlungsempfehlungen und ihre Adressatinnen (FF5)}

Im letzten Analyseschritt betrachten wir die Handlungsempfehlungen, in denen sich Normativität besonders stark manifestiert und mit denen (zum Teil) ein Transfer aus der Kommunikationswissenschaft in die Öffentlichkeit angestrebt wird. Untersucht wird, wie häufig Handlungsempfehlungen Teil einer Sollensvorstellung sind und an wen sie sich richten. Insgesamt enthält nur knapp jede dritte Sollensvorstellung (29\%) eine Handlungsempfehlung (Deutschland: 30\%; USA: 28\%; $\chi^{2}$ (1, $N=3233)=0,971$; n.s.), was für eine eher geringe Normativität in dieser Hinsicht spricht. An die Wissenschaft (Deutschland: 28\%, Rang 2; USA: 26\%, Rang 2) und an die Gesellschaft (Deutschland: 5\%, Rang 4; USA: 5\%, Rang 5) richten Autorinnen unabhängig von ihrer Herkunft gleich häufig Handlungsempfehlungen. Abgesehen davon unterscheiden sich die Adressatinnen der Handlungsempfehlun- 
gen signifikant zwischen beiden Samples - und wieder zeigt sich die erheblich größere Bedeutung des Mediensystems als Bezugspunkt normativer Äußerungen in Deutschland (Abb. 6): An die Medienpraxis richten deutsche Autorinnen ihre Handlungsempfehlungen (44\%, Rang 1) gut doppelt so häufig wie US-amerikanische (20\%, Rang 3). Letztere zielen öfter auf individuelle Akteurinnen (USA: $27 \%$, Rang 1; Deutschland: 5\%, Rang 4), Politik/Gesetzgebung (USA: 11\%, Rang 4; Deutschland: 7\%, Rang 3) und die Wirtschaft (USA: 4\%, Rang 6; Deutschland: $1 \%$, Rang 6).

\section{Diskussion}

Die vorliegende Studie untersucht eine bislang vernachlässigte Frage: Unterscheidet sich die Normativität von Fachzeitschriftenaufsätzen in Deutschland und den USA, und inwiefern könnten etwaige Unterschiede auf unterschiedliche strukturelle Einflüsse in den beiden Ländern zurückgeführt werden? Als Messinstrument für ihre Beantwortung schlagen wir die Konzepte der Sollensvorstellungen - bestehend aus den drei Elementen Inhalt, Subjekt und Objekt - und Handlungsempfehlungen vor und untersuchen deren Vorkommen in Aufsätzen, die zwischen 1970 und 2014 in deutschen und internationalen Fachzeitschriften erschienen sind.

Die Ergebnisse bestätigen die stark normative Prägung der deutschen wie auch der US-amerikanischen Kommunikationswissenschaft: Fast neun von zehn Aufsätzen enthalten Sollensvorstellungen (FF1). Auch hinsichtlich weiterer formaler Indikatoren unterscheiden sich beide Länder kaum: Sowohl in Deutschland als auch in den USA werden erstrebenswerte Zustände für die Zukunft deutlich häufiger beschrieben, als dass aktuelle Gegebenheiten bewertet werden (FF2). Die Autorinnen beziehen mehrheitlich selbst normativ Position, statt Aussagen anderer Urheberinnen zu zitieren $(F F 4)$. Nur etwa jede dritte Sollensvorstellung in beiden Ländern benennt ein Subjekt, das den wünschenswerten Zustand herstellen soll, jede fünfte Sollensvorstellung erwähnt ein Objekt, das von diesem Zustand profitieren soll $(F F 3)$, und jede dritte wird mit einer Handlungsempfehlung verbunden $(F F 5)$.

Die Indikatoren, die sich stärker auf die Inhalte der normativen Äußerungen richten, offenbaren jedoch deutliche Länderunterschiede. In Hinblick auf die übergeordnete Forschungsfrage legen unsere Befunde den Schluss nahe, dass strukturelle Einflüsse normative Äußerungen im Fach prägen: Sowohl als Inhalte, Subjekte und Objekte der Sollensvorstellungen $(F F 3)$ wie auch als Adressatinnen der Handlungsempfehlungen (FF5) dominieren in Deutschland das Mediensystem und seine Akteurinnen, die in den USA gleichrangig mit oder sogar nachrangig hinter der Gesellschaft und ihren Akteurinnen sind. Dafür gibt es verschiedene denkbare Gründe. Einer davon ist die Zugehörigkeit zu unterschiedlichen Typen von Mediensystemen. Deutschland ist ein demokratisch-korporatistisches Land (vgl. Hallin und Mancini 2004, S. 67), in dem sich hohe normative Erwartungen an die Medien (den öffentlich-rechtlichen Rundfunk, aber auch die Presse mit ihrer öffentlichen Aufgabe) richten. Inwieweit die Medien diese Erwartungen erfüllen, ist in Deutschland eine hochrelevante, normative und gesellschaftlich wie politisch kontrovers diskutierte Frage im Untersuchungszeitraum, z. B. vor dem Hintergrund der wachsenden 
Pressekonzentration, der Dualisierung des Rundfunks und der immer wieder aufflammenden Parteilichkeitsvorwürfe an den öffentlich-rechtlichen Rundfunk. Die gesellschaftliche und politische Relevanz solcher Fragen - und dass die Politik und Drittmittelgeber wie die Landesmedienanstalten in Deutschland entsprechende Studien nachfragen - beeinflusst die Themen kommunikationswissenschaftlicher Forschung. All dies bestimmt die Medien als zentralen Kontext, innerhalb dessen deutsche Kommunikationswissenschaftlerinnen sich normativ äußern, was sich auch in ihren wissenschaftlichen Publikationen spiegelt. Davon zeugt auch der besondere Schwerpunkt deutscher Sollensvorstellungen auf Medienpolitik und Qualitätssicherung sowie auf verschiedene Normen und Werte, die mit der öffentlichen Aufgabe der Medien in Verbindung stehen: Objektivität, Neutralität, Bildung, Informiertheit, soziale Integration und Inklusion.

In den USA mit ihrem liberalen Mediensystem spielt der öffentlich-rechtliche Rundfunk eine untergeordnete Rolle. Die Medien werden vor allem durch Wettbewerb reguliert, davon ausgehend, dass die ökonomischen Marktmechanismen - Angebot und Nachfrage - ein ,ideales Medienangebot“" gewährleisten. Diese ökonomische Ausrichtung der Medien erklärt auch, warum US-amerikanische Sollensvorstellungen mehr Wert auf das Wecken von Aufmerksamkeit legen (vgl. Magin 2019, S. 1707). Der höhere Stellenwert der Gesellschaft als Bezugspunkt von Normativität hängt damit zusammen, dass das US-Wissenschaftssystem stärker als das deutsche danach strebt, ,mit Hilfe wissenschaftlicher Arbeit die Welt zu verbessern“ (Meyen 2013, S. 118). Damit einher geht die Absicht, alle Bereiche menschlichen Lebens zu erreichen, z.B. auch das Gesundheitswesen, das in den US-amerikanischen Sollensvorstellungen erheblich präsenter ist als in den deutschen. Dass sich US-Autorinnen in ihren Handlungsempfehlungen dreimal so häufig wie deutsche an individuelle Akteurinnen wie Eltern oder Patientinnen richten, dürfte mit der stark individualistischen Kultur der USA (vgl. Hofstede 2001, S. 249) zusammenhängen, die das „Ich“ in den Mittelpunkt stellt.

Zugleich spiegeln die Befunde die unterschiedlichen Fachentwicklungen diesund jenseits des Atlantiks wider: Die deutsche Kommunikationswissenschaft war von Beginn an eng mit der Journalistinnenausbildung verbunden. Journalisten wurden auf Professuren berufen und waren Mitglieder medienpolitischer Fachkommissionen und Arbeitsgemeinschaften (vgl. Meyen und Wendelin 2008, S. 11). In den USA hingegen fußt das Fach neben Journalistik und Massenkommunikationsforschung auch auf der Traditionslinie Speech und Rhetoric (vgl. Meyen 2013, S. 117), worin wahrscheinlich der häufigere normative Bezug auf Konversationsnormen in US-Aufsätzen begründet ist.

Dass Subjekte und Objekte aus dem Gesundheitssystem und Sollensvorstellungen zur physischen und psychischen Gesundheit nahezu ausschließlich in den USAufsätzen vorkommen, resultiert mit hoher Wahrscheinlichkeit daraus, dass sich die Gesundheitskommunikation als Teildisziplin der Kommunikationswissenschaft in den USA deutlich früher herausgebildet und durch die Gründung eigenständiger wissenschaftlicher Zeitschriften und einer eigenen Sektion innerhalb der International Communication Association etabliert hat (vgl. Schulz und Hartung 2010; Spatzier und Signitzer 2014). Die Gesundheitskommunikation ist dort ein „Bereich der angewandten Kommunikationswissenschaft": Viele der verhandelten Forschungsfra- 
gen werden ,außerhalb der wissenschaftlichen Community“ (Spatzier und Signitzer 2014, S. 46) formuliert - ein erneuter Beleg für den engeren Bezug der USCommunity zu medienexternen Gesellschaftsbereichen.

\section{Limitationen, zukünftige Fragestellungen und normative Implikationen}

Wie jede wissenschaftliche Studie hat auch unsere Limitationen. Fachzeitschriftenaufsätze bilden das Fach nicht in seiner Gesamtheit ab. Wir verstehen unsere Studie daher als ersten Schritt, dem die Untersuchung weiterer Publikationsformen wie Monografien und Sammelbände folgen sollte. Diese sollten auch geografische Regionen wie Asien und Afrika in den Blick nehmen. Auch sollte untersucht werden, wie sich die Normativität des Fachs im Zeitverlauf entwickelt hat und ob das Publizieren in internationalen Fachzeitschriften zu einem Angleichungsprozess in normativer Hinsicht führt, unabhängig von der Nationalität der Autorinnen. Das ist mit den hier erhobenen Daten möglich, hätte aber den Rahmen dieses Aufsatzes gesprengt und soll daher Gegenstand weiterer Analysen sein. Gleiches gilt für den Zusammenhang zwischen den Subjekten und Objekten einer Sollensvorstellung und den Forschungsbereichen. Aussagen hierzu können wir aufgrund zu geringer Fallzahlen nicht treffen.

Wünschenswert wäre es, die Inhaltsanalyse kontinuierlich fortzusetzen, um aktuelle Einflüsse und normative Entwicklungen des Fachs beobachten zu können. Gerade der aktuelle Fachdiskurs zu den gesellschaftlichen Folgen der Tech-Intermediäre wie Facebook und Google bzw. zu neuen Plattform-Trends in der digitalen Ära (wie Platformization, Datafication und Algorithmen) dürfte stark normativ geprägt sein. Dies konnten wir aufgrund des Förderzeitraums unserer Studie nicht mehr berücksichtigen. Künftige Untersuchungen dazu sind aber wichtig und lohnenswert. Die vergleichende Untersuchung der Normativität unterschiedlicher Forschungsbereiche sollte in Folgestudien ausgeweitet und vertieft werden. Um noch besser zu verstehen, wie die Entstehungskontexte die normative Prägung wissenschaftlicher Publikationen beeinflussen, könnte eine Befragung der Autorinnen zu ihrer Sozialisation, der Wahrnehmung ihrer Scientific Community und ihren Norm- und Wertvorstellungen unsere Analyse sinnvoll ergänzen.

Trotz dieser Limitationen hat die vorliegende Untersuchung drei zentrale Stärken gegenüber bisherigen Versuchen, die Normativität der Kommunikationswissenschaft zu erfassen: Erstens liefert das vorgeschlagene Instrument der Sollensvorstellungen und Handlungsempfehlungen eine Möglichkeit, Normativität jenseits der Verwendung der Begriffe Normen und Werte sichtbar zu machen - in wissenschaftlichen Publikationen und darüber hinaus (z. B. in Untersuchungen zu normativen Äußerungen in Fernsehserien und Internetforen oder zu normativen Debatten über mediale Alltagspraktiken, vgl. Venema 2019). Das hier entwickelte methodische Design ermöglicht zweitens nicht nur systematische Vergleiche über einzelne Teilbereiche des Fachs oder Forschungsgegenstände hinweg, sondern auch zwischen verschiedenen Fachkulturen. Damit leistet das Konzept der Manifestationen von Normativität drittens einen wichtigen Beitrag, welcher der Selbstreflexion der Kommunikations- 
wissenschaft und seiner Normativität dienlich sein kann: Der Vergleich führt vor Augen, wie stark das wissenschaftliche Arbeiten durch die umgebenden Strukturen geprägt ist. Insbesondere welche Forschungsgegenstände als relevant empfunden und welche normativen Positionen vertreten werden, hängt stark davon ab, in welchem nationalen und kulturellen Kontext Wissenschaftlerinnen beruflich sozialisiert werden und arbeiten. Diese Erkenntnis bewahrt vor unzulässigen Verallgemeinerungen eigener Erfahrungen und des eigenen Relevanzempfindens. Gleichzeitig ermöglichen vergleichende Studien wie diese, besser zu verstehen und zu erklären, welche Faktoren die normativen Positionen von Wissenschaftlerinnen beeinflussen (siehe auch Melischek et al. 2008, S. 10-11).

Mit diesen Erkenntnissen können Wissenschaftlerinnen besser begründet entscheiden, ob und in welcher Form sie die normativen Bezüge, die ihrer Forschung und Lehre zugrunde liegen, explizit(er) machen sowie in öffentlichen Debatten thematisieren wollen. Aktuelle Diskussionen im Fach greifen die Diskussion um die (Un-)Sichtbarkeit der Kommunikationswissenschaft in öffentlichen Diskursen (wieder) auf (vgl. z. B. Borchardt 2019; Öffentliche Kommunikationswissenschaft 2019; Pörksen 2015). Freilich muss sich nicht jede Kommunikationswissenschaftlerin am öffentlichen Diskurs beteiligen, sondern kann sich auch ausschließlich als Beobachterin der Gesellschaft verstehen. Doch Wissenschaftlerinnen, welche die Fragen der Gesellschaft beantworten, kritische Debatten anstoßen (vgl. Weichert 2011) und Orientierung für medienbezogenes Handeln bieten wollen, können das auf einer solideren Grundlage tun, wenn sie sich ihrer normativen Standpunkte bewusst sind und auch wissen, wodurch diese beeinflusst werden. Dieses Bewusstsein ermöglicht auch, normative Äußerungen explizit und reflektiert $\mathrm{zu}$ formulieren - in wissenschaftlichen Publikationen und darüber hinaus.

Funding Open Access funding enabled and organized by Projekt DEAL.

Open Access Dieser Artikel wird unter der Creative Commons Namensnennung 4.0 International Lizenz veröffentlicht, welche die Nutzung, Vervielfältigung, Bearbeitung, Verbreitung und Wiedergabe in jeglichem Medium und Format erlaubt, sofern Sie den/die ursprünglichen Autor(en) und die Quelle ordnungsgemäß nennen, einen Link zur Creative Commons Lizenz beifügen und angeben, ob Änderungen vorgenommen wurden.

Die in diesem Artikel enthaltenen Bilder und sonstiges Drittmaterial unterliegen ebenfalls der genannten Creative Commons Lizenz, sofern sich aus der Abbildungslegende nichts anderes ergibt. Sofern das betreffende Material nicht unter der genannten Creative Commons Lizenz steht und die betreffende Handlung nicht nach gesetzlichen Vorschriften erlaubt ist, ist für die oben aufgeführten Weiterverwendungen des Materials die Einwilligung des jeweiligen Rechteinhabers einzuholen.

Weitere Details zur Lizenz entnehmen Sie bitte der Lizenzinformation auf http://creativecommons.org/ licenses/by/4.0/deed.de.

\section{Literatur}

Albert, H. (1993). Wertfreiheit als methodisches Prinzip. Zur Frage der Notwendigkeit einer normativen Sozialwissenschaft. In E. Topitsch (Hrsg.), Logik der. Sozialwissenschaften, (Bd. 10, S. 196-225). Königstein: Kiepenheuer \& Witsch. 
Althaus, S.L. (2012). What's good and bad in political communication research? Normative standards for evaluating media and citizen performance. In H. A. Semetko \& M. Scammell (Hrsg.), The SAGE Handbook of Political Communication (S. 97-112). London: SAGE.

Anderson, M.S., Ronning, E. A., De Vries, R., \& Martinson, B.C. (2010). Extending the Mertonian norms: scientists' subscription to norms of research. The Journal of Higher Education, 81, 366-393.

Averbeck-Lietz, S. (2012). Communication studies beyond the national: Connections and disconnections between research communities and how to study them. Global Media Journal, 2(2), 1-10.

Beauchamp, T.L., \& Childress, J.F. (2013). Principles of biomedical ethics (7. Aufl.). Oxford: Oxford University Press.

Beck, K. (2020). Kommunikationswissenschaft (6., überarb. Aufl.). Konstanz: UVK.

Blumler, J.G., \& Cushion, S. (2014). Normative perspectives on journalism studies. Stock-taking and future directions. Journalism, 15, 259-272.

Borchardt, A. (2019). Verletzter Stolz und Vorurteile. Aviso, o.J.(68), 12-13.

Boudana, S. (2011). A definition of journalistic objectivity as a performance. Media, Culture \& Society, $33,385-398$.

Boventer, H. (1984). Ethik des Journalismus. Zur Philosophie der Medienkultur. Konstanz: UVK.

Braxton, J.M. (2010). Norms and the work of colleges and universities: introduction to the special issue-Norms in academia. The Journal of Higher Education, 81, 243-250.

Brüggemann, M., Engesser, S., Büchel, F., Humprecht, E., \& Castro, L. (2014). Hallin and Mancini revisited: four empirical types of western media systems. Journal of Communication, 6, 1037-1065.

Bruhn, J.G. (2008). Value dissonance and ethics failure in academia: a causal connection? Journal of Academic Ethics, 6, 17-32.

Chesebro, J. W. (2003). Communication, values, and popular television series-a twenty-five year assessment and final conclusions. Communication Quarterly, 51, 367-418.

Christians, C., Glasser, T. L., McQuail, D., Nordenstreng, K., \& White, R. A. (2009). Nomative theories of the media: journalism in democratic societies. University of Illinois Press: Urbana.

Dahrendorf, R. (1968). Pfade aus Utopia. Arbeiten zu Theorie und Methode der Soziologie. Gesammelte Abhandlungen I. München: Piper.

DGPuK (2008). Kommunikation und Medien in der Gesellschaft: Leistungen und Perspektiven der Kommunikations- und Medienwissenschaft. Eckpunkte für das Selbstverständnis der Kommunikationsund Medienwissenschaft. Selbstverständnispapier der Deutschen Gesellschaft für Publizistik- und Kommunikationswissenschaft (DGPuK). https:/www.dgpuk.de/de/selbstverst\%C3\%A4ndnis-derdgpuk.html. Zugegriffen: 20. Nov. 2020.

Eberwein, T., \& Fengler, S. (2012). Theorie und Praxis in der Kommunikations- und Medienforschung. Einführung und Überblick. In T. Eberwein, S. Fengler \& J. Jorch (Hrsg.), Theoretisch praktisch!? Anwendungsoptionen und gesellschaftliche Relevanz der Kommunikations- und Medienforschung (S. 11-26). Konstanz: UVK.

Esser, H. (2000). Soziologie. Spezielle Grundlagen. Bd. 5: Institutionen. Frankfurt am Main: Campus.

Feng, G. C. (2013). Underlying determinants driving agreement among coders. Quality and Quantity, 47, 2983-2997.

Fink, E. J., \& Gantz, W. (1996). A content analysis of three mass communication research traditions: social science, interpretive studies, and critical analysis. Journalism \& Mass Communication Quarterly, 73, $114-134$.

Foss, K. A. (2008). „You're gonna make it after all“" changing cultural norms as described in the lyrics of sitcom theme songs, 1970-2001. Rocky Mountain Communication Review, 5, 43-56.

Früh, W. (2001). Inhaltsanalyse: Theorie und Praxis (5., überarb. Aufl.). Konstanz: UVK.

Gehrau, V., Röttger, U., \& Schulte, J. (2013). Reputation von Hochschulen. Erwartungen, Anforderungen und mediale Informationsquellen von Studierenden. In U. Röttger, V. Gehrau \& J. Preusse (Hrsg.), Strategische Kommunikation. Umrisse und Perspektiven eines Forschungsfeldes (S. 323-344). Wiesbaden: Springer.

Gerholm, T. (1990). On tacit knowledge in academia. European Journal of Education, 25, 263-271.

Hallin, D.C., \& Mancini, P. (2004). Comparing media systems. Three models of media and politics. Cambridge: Cambridge University Press.

Harnischmacher, M. (2010). Journalistenausbildung im Umbruch. Zwischen Medienwandel und Hochschulreform: Deutschland und USA im Vergleich. Konstanz: UVK.

Hasebrink, U. (2016). Der individuelle Wert von Medienangeboten in digitalen Medienumgebungen. In ORF (Österreichischer Rundfunk) (Hrsg.), Public Social Value (S. 4-35). Wien: ORF.

Heesen, J. (Hrsg.). (2016). Handbuch Medien- und Informationsethik. Stuttgart: J. B. Metzler. 
Herbst, S. (2008). Disciplines, intersections, and the future of communication research. Journal of Communication, 58, 603-614.

Hofstede, G. (2001). Culture's consequences. Comparing values, behaviors, institutions and organizations across nations. Thousand Oaks: SAGE.

Howaldt, J. (2005). Die Soziologie in Zeiten der Wissensgesellschaft: Kritische Anmerkungen zu einer unzeitgemäßen Unterscheidung. Sozialwissenschaften und Berufspraxis, 28, 186-201.

Joas, H. (1997). Die Entstehung der Werte. Frankfurt am Main: Suhrkamp.

Kepplinger, H. M. (2014). Normative theories of political communication. In C. Reinemann (Hrsg.), Political communication (S. 21-38). Berlin: De Gruyter Mouton.

Kepplinger, H. M., \& Post, S. (2008). Der Einfluss der Medien auf die Klimaforschung. Natur \& Geist, 24, 25-28.

Krippendorff, K. (2008). Reliability. In W. Donsbach (Hrsg.), The international encyclopedia of communication. https://doi.org/10.1002/9781405186407.wbiecr029.

Kuhn, T. (1962). The structure of scientific revolutions. Chicago: University of Chicago Press.

Lang, A. (2013). Discipline in crisis? The shifting paradigm of mass communication research. Communication Theory, 23, 10-24.

Lasswell, H. D. (1948). The structure and function of communication in society. In L. Bryson (Hrsg.), The communication of ideas (S. 37-51). New York: Cooper Square.

Lenhardt, G. (2005). Hochschulen in Deutschland und in den USA. Deutsche Hochschulpolitik in der Isolation. Wiesbaden: Springer.

Löblich, M. (2010a). Die empirisch-sozialwissenschaftliche Wende in der Publizistik- und Zeitungswissenschaft. Köln: Halem.

Löblich, M. (2010b). Die empirisch-sozialwissenschaftliche Wende. Ein Beitrag zur historischen und kognitiven Identität der Kommunikationswissenschaft. Medien \& Kommunikationswissenschaft, 58, 544-562.

Löblich, M., \& Scheu, A. (2011). Writing the history of communication studies: a sociology of science approach. Communication Theory, 21, 1-22.

Magin, M. (2019). Attention, please! Structural influences on tabloidization of campaign coverage in German and Austrian elite newspapers (1949-2009). Journalism, 20, 1704-1724.

Magin, M. Politischer Parallelismus. In M. Prinzing \& R. Blum (Hrsg.), Handbuch politischer Journalismus. Köln: Halem. im Erscheinen.

McQuail, D. (1992). Media Performance. Mass communication and the public interest. London: SAGE.

Melischek, G., Seethaler, J., \& Wilke, J. (2008). Einführung. In G. Melischek, J. Seethaler \& J. Wilke (Hrsg.), Medien \& Kommunikationsforschung im Vergleich. Grundlagen, Gegenstandsbereiche, Verfahrensweisen (S. 9-16). Wiesbaden: VS.

Merton, R. K. (1973). The sociology of science. Theoretical and empirical investigations. Chicago: University of Chicago Press.

Meyen, M. (2013). Normativität in der US-Community. Ein Beitrag zu den Strukturen des kommunikationswissenschaftlichen Feldes. In M. Karmasin, M. Rath \& B. Thomaß (Hrsg.), Normativität in der Kommunikationswissenschaft (S. 117-132). Wiesbaden: Springer.

Meyen, M., \& Wendelin, M. (2008). Wissenschaftliche Autonomie und gesellschaftlicher Bedarf. Eine Einführung. In M. Meyen \& M. Wendelin (Hrsg.), Journalistenausbildung, Empirie und Auftragsforschung (S. 7-27). Köln: Halem.

Neuberger, C. (2017a). Erwartungen der Gesellschaft an das Internet und ihre Erfüllung. Zur Begründung und Anwendung normativer Maßstäbe. Medien Journal, , 2, 45-60.

Neuberger, C. (2017b). Journalistische Objektivität. Vorschlag für einen pragmatischen Theorierahmen. Medien und Kommunikationswissenschaft, 65, 406-431.

Öffentliche Kommunikationswissenschaft (2019). Charta Öffentliche Kommunikationswissenschaft. https://oeffentliche-kowi.org/charta/. Zugegriffen: 20. Nov. 2020.

Peters, H.P. (2019). WissenschaftlerInnen als Kommunikatoren. In B. Fähnrich, J. Metag, S. Post \& M.S. Schäfer (Hrsg.), Forschungsfeld Hochschulkommunikation (S. 209-225). Wiesbaden: Springer.

Pörksen, B. (2015). Wo seid ihr, Professoren? Die Zeit. https://www.zeit.de/2015/31/wissenschaftprofessoren-engagement-oekonomie. Zugegriffen: 2. Apr. 2020.

Post, S. (2013). Wahrheitskriterien von Journalisten und Wissenschaftlern. Baden-Baden: Nomos.

Rath, M. (2010). Empirische Perspektiven. In C. Schicha \& C. Brosda (Hrsg.), Handbuch Medienethik (S. 136-146). Wiesbaden: VS.

Redelfs, M. (1996). Investigative Reporting in den USA. Strukturen eines Journalismus der Machtkontrolle. Opladen: Westdeutscher Verlag. 
Schäfers, B. (2016). Lektion II. Soziales Handeln und seine Grundlagen: Normen, Werte, Sinn. In H. Korte \& B. Schäfers (Hrsg.), Einführung in Hauptbegriffe der Soziologie (9. Aufl.) (S. 24-48). Wiesbaden: Springer.

Scherer, H. (2013). Normativität in der quantitativen empirischen Kommunikationswissenschaft. In M. Karmasin, M. Rath \& B. Thomaß (Hrsg.), Normativität in der Kommunikationswissenschaft (S. 247-265). Wiesbaden: Springer.

Schicha, C. (2010). Philosophische Ethik. In C. Schicha \& C. Brosda (Hrsg.), Handbuch Medienethik (S. 21-40). Wiesbaden: VS.

Schulz, P. J., \& Hartung, U. (2010). Health communication research in europe: an emerging field. Health Communication, 25, 548-551.

Serong, J. (2011). Public Value im Internet und Drei-Stufen-Test. In H. Gundlach (Hrsg.), Public Value in der Digital- und Internetökonomie (S. 101-120). Köln: Halem.

Singer, J. B., Domingo, D., Heinonen, A., Hermida, A., Paulussen, S., Quandt, T., Reich, Z., \& Vujnovic, M. (Hrsg.). (2011). Participatory journalism: Guarding open gates at online newspapers. Oxford: Wiley-Blackwell.

Soffer, O., \& Geifman, D. (2020). Comparing research topics in European and International Communication Association journals: computational analysis. The International Communication Gazette. https:// doi.org/10.1177/1748048520928334.

Sommer, U. (2016). Werte. Warum man sie braucht, obwohl es sie nicht gibt. Stuttgart: J. B. Metzler.

Spatzier, A., \& Signitzer, B. (2014). Ansätze und Forschungsfelder der Gesundheitskommunikation. In K. Hurrelmann \& E. Baumann (Hrsg.), Handbuch Gesundheitskommunikation (S. 34-50). Bern: Huber.

Thomaß, B. (2016). Medien- und Kommunikationswissenschaft. In J. Heesen (Hrsg.), Handbuch Medienund Informationsethik (S. 33-40). Stuttgart: J. B. Metzler.

Venema, R. (2019). Bilder, Normen, Diskurse: Theoretisch-konzeptionelle Grundlagen für die Analyse von Normen in Debatten über visuelle Alltagspraktiken. In C. Schwender, C. Brantner, C. Graubner \& J. von Gottberg (Hrsg.), zeigen - andeuten - verstecken: Bilder zwischen Verantwortung und Provokation (S. 40-59). Köln: Halem.

Vogler, D., \& Post, S. (2019). Reputation von Hochschulen. In B. Fähnrich, J. Metag, S. Post \& M. S. Schäfer (Hrsg.), Forschungsfeld Hochschulkommunikation (S. 319-340). Wiesbaden: Springer.

Waisbord, S. (2016). Communication studies without frontiers? Translation and cosmopolitanism across academic cultures. International Journal of Communication, 10, 868-886.

Weichert, S. (2011). „Aufklärung bleibt auf der Strecke“. Stephan Weichert über Self-Marketing und Machtspielchen im Fach. Aviso, o.J.(53), 8-9.

Zillich, A.F., Riesmeyer, C., Magin, M., Müller, K.F., Pfaff-Rüdiger, S., Rothenberger, L., \& Sehl, A. (2016). Werte und Normen als Sollensvorstellungen in der Kommunikationswissenschaft - ein Operationalisierungsvorschlag. Publizistik, 61, 393-411.

PD Dr. Stephanie Geise ist wissenschaftliche Mitarbeiterin am Institut für Kommunikationswissenschaft der Westfälischen Wilhelms-Universität Münster.

Dr. Ulrike Klinger ist Professorin für Politische Theorie und Digitale Demokratie an der Europa-Universität Viadrina Frankfurt (Oder).

Dr. Melanie Magin ist Associate Professorin in Mediensoziologie an der Norwegian University of Science and Technology (NTNU) in Trondheim.

Dr. Kathrin Friederike Müller ist wissenschaftliche Mitarbeiterin am Institut für Medienforschung der Universität Rostock.

Dr. Cordula Nitsch ist wissenschaftliche Mitarbeiterin an der Professur für Kommunikationswissenschaft - Öffentliche Kommunikation der Universität Augsburg.

Dr. Claudia Riesmeyer ist Akademische Oberrätin auf Lebenszeit am Institut für Kommunikationswissenschaft und Medienforschung der Ludwig-Maximilians-Universität München.

PD Dr. Liane Rothenberger ist Akademische Oberrätin am Institut für Medien und Kommunikationswissenschaft der Technischen Universität Ilmenau. 
Dr. Christina Schumann ist wissenschaftliche Mitarbeiterin am Institut für Medien und Kommunikationswissenschaft der Technischen Universität Ilmenau.

Dr. Annika Sehl ist Professorin für Digitalen Journalismus an der Universität der Bundeswehr München.

Dr. Cornelia Wallner ist Akademische Rätin am Institut für Kommunikationswissenschaft und Medienforschung der Ludwig-Maximilians-Universität München.

Dr. Arne Freya Zillich ist Akademische Mitarbeiterin an der Filmuniversität Babelsberg KONRAD WOLF. 